\title{
Thermal Conductivity of Cementitious Composites \\ Containing Microencapsulated Phase Change Materials
}

Alex Ricklefs ${ }^{1}$, Alexander M. Thiele ${ }^{1}$, Gabriel Falzone ${ }^{2}$, Gaurav Sant ${ }^{2,3}$, and Laurent Pilon ${ }^{1,+}$

\author{
University of California, Los Angeles \\ Henry Samueli School of Engineering and Applied Science \\ ${ }^{1}$ Mechanical and Aerospace Engineering Department \\ ${ }^{2}$ Civil and Environmental Engineering Department \\ Laboratory for the Chemistry of Construction Materials (LC $\left.{ }^{2}\right)$ \\ ${ }^{3}$ California Nanosystems Institute (CNSI) \\ +Corresponding Author: Phone: +1 (310)-206-5598, Fax: +1 (310)-206-2302 \\ E-mail: pilon@seas.ucla.edu
}

March 12, 2016 


\section{Abstract}

This paper investigates the effects of adding microencapsulated phase change materials (PCM) on the thermal conductivity of cement paste and cement mortar composites. Embedding cementitious composites with microencapsulated PCM has been considered a promising method for increasing the thermal mass of buildings to achieve greater energy efficiency and for reducing the risks of thermal cracking in pavements. Cement paste and cement mortar samples were synthesized with a constant water to cement ratio of 0.45 . Both contained microencapsulated PCM with diameter ranging from 17-20 $\mu \mathrm{m}$, volume fraction up to $30 \%$, and a melting temperature around $24^{\circ} \mathrm{C}$. The cement mortar also contained quartz grains 150-600 $\mu \mathrm{m}$ in diameter such that the sum of the volume fractions of quartz and microencapsulated PCM was fixed at 55\%. All samples were aged for more than 28 days. Their effective density and free moisture content were systematically measured. A guarded hot plate apparatus was designed, assembled, and validated according to the ASTM C177-13 to measure the effective thermal conductivity of the aged specimens of cement paste and cement mortar without and with microencapsulated PCM. Measurements were performed between 10 and $40^{\circ} \mathrm{C}$, encompassing the entire PCM phase change temperature window. The effective thermal conductivity of both the cement paste and the cement mortar composites was found to be nearly independent of temperature in the range considered. It also decreased as the volume fraction of microencapsulated PCM increased. Finally, excellent agreement was obtained between experimental data and the effective medium approximation derived by Felske (2004) for core-shell-matrix composites. These results can be used to design cementitious composite materials containing microencapsulated PCMs for energy efficient buildings and crack-resistant pavements.

Keywords: Thermal conductivity, phase change materials, microencapsulated, cement, mortar, core-shell-matrix composites 


\section{NOMENCLATURE}

$\begin{array}{ll}A_{g a p} & \text { area of the gap between the metered and guard heaters, } \mathrm{mm}^{2} \\ A_{m} & \text { area of the metered heater, } \mathrm{mm}^{2} \\ c_{p} & \text { specific heat, } \mathrm{J} /(\mathrm{kg} \cdot \mathrm{K}) \\ e & \text { thermal effusivity, } \mathrm{W} \cdot \mathrm{s}^{1 / 2} /\left(\mathrm{m}^{2} \cdot \mathrm{K}\right) \\ k & \text { thermal conductivity, } \mathrm{W} /(\mathrm{m} \cdot \mathrm{K}) \\ L_{i} & \text { vertical distance between thermocouples in sample } i, \mathrm{~m} \\ m & \text { mass, } \mathrm{g} \\ \dot{m} & \text { coolant mass flow rate, } \mathrm{g} / \mathrm{s} \\ q_{m} & \text { heat transfer rate from the metered heater, } \mathrm{W} \\ q_{l o s s} & \text { estimated heat loss, W } \\ q_{w, i} & \text { heat transfer rate removed by cold plate } i, \mathrm{~W} \\ q_{i}^{\prime \prime} & \text { incoming heat flux in sample } i, \mathrm{~W} / \mathrm{m}^{2} \\ \dot{Q}_{i} & \text { coolant volumetric flow rate, mL } / \text { min } \\ R_{g} & \text { volume, mm }{ }^{3} \\ R_{m} & \text { vosistance of the guard heater wire, } \Omega \\ T_{j, i} & \text { resistance of the metered heater wire, } \Omega \\ U_{g} & \text { temperature measured by thermocouple } j \text { in sample } i,{ }^{\circ} \mathrm{C} \\ U_{m} & \text { voltage across the metered heater wire, } \mathrm{V} \\ w / c & \end{array}$

\section{Greek symbols}

$\alpha$

$\Delta x$

$\phi_{p}$

$\phi_{c+s}$ thermal diffusivity, $\mathrm{m}^{2} / \mathrm{s}$

associated systematic error in measurement $x$

volume fraction of phase $p$ in the cementitious composite

volume fraction of microcapsules in the cementitious composite, $\phi_{c+s}=\phi_{c}+\phi_{s}$ 


$\begin{array}{ll}\phi_{c / s} & \text { volume fraction of core in the microcapsule, } \phi_{c / s}=\phi_{c} /\left(\phi_{c}+\phi_{s}\right) \\ \phi_{w} & \text { free moisture content } \\ \rho & \text { density, } \mathrm{g} / \mathrm{cm}^{3} \\ \sigma_{q} & \text { standard deviation of the heat transfer rate } q_{i}, \mathrm{~W}\end{array}$

\section{Subscripts}

c

$c+s$

cold, $i$

$d r y$

eff

$i$

$m$

$q$

S

sat

$w$

$w, 1, i$

$w, 2, i$ refers to core

refers to core-shell particle

refers to the cold plate in contact with sample $i$

refers to fully dry free moisture content

refers to effective properties

refers to sample A or B

refers to matrix

refers to quartz sand

refers to shell

refers to fully saturated free moisture content

refers to the chiller coolant

refers to chiller coolant entering cold plate $i$

refers to chiller coolant exiting cold plate $i$ 


\section{Introduction}

Embedding phase change materials (PCMs) in a concrete matrix has been proposed as a mean to improve building energy efficiency [1-3] and reduce the risk of thermal cracking $[4,5]$. PCMs achieve such beneficial actions by increasing the thermal mass (i.e., heat capacity) and thermal resistance by lowering the effective thermal conductivity of the cementitious composite materials [1-3]. Indeed, PCMs can store and release thermal energy in the form of latent heat through a reversible phase transition between solid and liquid states, actions which superimpose onto the sensible heat capacity of the concrete [6-9].

The thermophysical properties of cementitious composites, such as the heat capacity and thermal conductivity, are critical input variables required for modeling their thermal behavior and engineering performance for design purposes. For example, these properties determine the heat flow into and out of the building as well as the time lag in the building thermal load $[10,11]$. Similarly, it is important to understand the development of temperature and restrained stress gradients in cementitious composites to estimate the risk of thermal cracking $[4,5]$. From thermodynamic considerations, the effective volumetric heat capacity of cementitious composite materials with embedded microencapsulated PCM can be express as [10]

$$
\left(\rho c_{p}\right)_{e f f}(T)=\phi_{c}\left(\rho c_{p}\right)_{c}(T)+\phi_{s}\left(\rho c_{p}\right)_{s}+\left(1-\phi_{c}-\phi_{s}\right)\left(\rho c_{p}\right)_{m}
$$

where $\phi_{c}$ and $\phi_{s}$ are the volume fractions of the core and shell materials, while $\left(\rho c_{p}\right)_{c},\left(\rho c_{p}\right)_{s}$, and $\left(\rho c_{p}\right)_{m}$ are the volumetric heat capacities of the core, shell, and matrix materials, respectively. On the other hand, the effect of PCM on the thermal conductivity of cementitious composites is non-trivial and limited reports are available $[1-3,7,12]$.

This paper aims to quantify the effect of incorporating microencapsulated PCM inclusions on the effective thermal conductivity of Type I ordinary Portland cement (OPC) pastes and mortars. A guarded hot plate apparatus was designed and fabricated, and its measurement performance rigorously validated. The effective thermal conductivity of cement pastes and 
mortars with various volume fractions of cement paste, mineral aggregates (e.g., quartz inclusions), and functional inclusions (microencapsulated PCMs) was measured. The results also provided experimental validation of effective medium approximation (EMAs) for threephase cementitious composites.

\section{Background}

\subsection{Thermal conductivity measurement methods}

Experimental methods available to measure the thermal conductivity of bulk solid materials can be categorized as either transient or steady-state. Transient methods include the plane source [13], hot strip [14], hot wire [15], hot bridge [16], and laser flash methods [17]. The first four methods involve temperature measurements collected over a time period ranging from $10 \mathrm{~ns}$ to $100 \mathrm{~s}$ during which the sample is heated [14-16]. A thin sensor is used to generate a pulse of thermal energy dissipated by the sample while simultaneously measuring the associated change in temperature at the sample surface [14-16]. The measured rate of thermal dissipation and change in sample temperature are used to calculate the thermal

effusivity defined as $e=\sqrt{\rho c_{p} k}$ where $\rho, c_{p}$, and $k$ are density, specific heat, and thermal conductivity of the sample, respectively [18]. The hot bridge method offers greater accuracy than the other three methods by using multiple sensors aligned in a Wheatstone bridge to collect sample surface temperature measurements with enhanced sensitivity [16]. Alternatively, the flash method [17] infers the thermal diffusivity $\alpha$, defined as $\alpha=k / \rho c_{p}$. A pulse of thermal energy is applied to the front face of a parallel plane sample while the change in temperature on the back face is measured over a period of time [17]. The thermal diffusivity is then calculated based on the thickness of the sample and the time required for the back surface to reach half of its maximum temperature measured over the duration of testing [17]. Since these transient methods do not measure thermal conductivity directly, uncertainty is introduced if the density $\rho$ and specific heat $c_{p}$ are not measured independently [19].

Steady-state methods, such as the hot plate and the guarded hot plate methods, measure 
the temperature difference across a sample maintained between a hot and a cold surface and subjected to one-dimensional (1D) steady-state heat conduction [20]. The thermal conductivity of the sample is determined from Fourier's law, based on the imposed heat flux and the measured temperature gradient across the sample [20,21]. The hot plate method features simple measurement and analysis $[20,21]$. However, radial heat losses in the hot plate make it difficult to achieve 1D steady-state conditions, thus introducing uncertainty in the measured thermal conductivity [20]. To mitigate these heat losses and to ensure 1D heat conduction, the guarded hot plate method includes a heated "guard" ring concentric to the center "metered" section of the heating element [20,21]. The gap is filled with either air or a thermally insulating material to enhance radial thermal resistance around the hot plate. In addition, the guard ring is maintained at the same temperature as the metered section.

Overall, steady-state methods offer a direct measurement of thermal conductivity $k$, whereas transient methods require prior knowledge of the sample's density $\rho$ and specific heat $c_{p}$. Steady-state methods are also simpler in terms of apparatus design and fabrication, experimental procedure, and data analysis [16]. However, they require longer experimental time than transient methods $[13,17,22]$. In this study, thermal conductivity measurements of cement paste and cement mortar containing microencapsulated PCM were performed using a guarded hot plate apparatus designed, fabricated, and validated per ASTM C177-13 [22].

\subsection{Thermal conductivity of cementitious composites}

Table 1 summarizes the effective thermal conductivity reported in the literature for cement paste with different water to cement ratio (w/c), age, and free moisture content $\phi_{w}$, defined as the ratio of the volume of free water in a composite to its total volume of porosity $[8,23-28]$. Most studies used one of the above-mentioned transient methods to measure the thermal conductivity. Xu and Chung [24] measured the thermal conductivity of Type I OPC paste samples with w/c of 0.45 using the laser flash method. More recently, Demirboga [26] measured the thermal conductivity of Type I OPC paste with a w/c of 0.35 using the hot wire method. Similarly, Bentz [23] measured the thermal conductivity of Type I OPC paste 
samples with w/c of 0.3 and 0.4 at $20^{\circ} \mathrm{C}$ using a transient plane source method. Table 1 indicates that measurements reported in these studies $[23,24,26]$ differed by a factor of 2 although they considered similar cement pastes. To the best of our knowledge, Demirboga [26] did not specify the sample temperature and specific heat while Bentz [23] did not specify the sample density. In addition, the specific heat of cement paste used by Xu and Chung [24] was $736 \mathrm{~J} /(\mathrm{kg} \cdot \mathrm{K})$ and almost half of that reported in the literature at $1530 \mathrm{~J} /(\mathrm{kg} \cdot \mathrm{K})[29]$. This probably explains why the thermal conductivity reported by $\mathrm{Xu}$ and Chung [24] was almost half of that reported by Demirboga [26] and Bentz [23].

The effective thermal conductivity of cement paste has been shown to decrease as the water to cement mass ratio increased [23]. This can be attributed to the enhanced pore formation within the composite with increasing amount of water [30]. The thermal conductivity of cement paste has also been observed to decrease by up to $30 \%$ over a 7 -day hardening period but remained unchanged beyond this period [31,32]. The hardening process can take place in either water, ambient air, or humid air with up to $100 \%$ humidity [31,32]. The thermal conductivity of cement paste was found to decrease significantly more when hardening occurred in ambient air [31] rather than in water [32], when the pores are filled with air instead of water. Kim et al. [33] found that the thermal conductivity of cement paste at temperatures between $20-60^{\circ} \mathrm{C}$ decreased by up to $35 \%$ as the free moisture content $\phi_{w}$ decreased from 1 to 0 . This can also be attributed to the difference in thermal conductivity between air and water filling up the pores [33].

Moreover, the type and volume fraction of aggregates present in cement mortar composites can alter their effective thermal conductivity $[25,33,34]$. Table 2 summarizes the experimental studies reporting the thermal conductivity of cement mortar with different types and amount of aggregate inclusions as well as w/c ratio, age, and free moisture content $\phi_{w}[25,28,33]$. For example, Uysal et al. [25] measured, using the hot wire method, the effective thermal conductivity of cement mortar containing pumice aggregate with a volume fraction $\phi_{a g g}$ of up to 0.75 . They found that the thermal conductivity was up to $46 \%$ lower than that of similar plain cement paste samples. This can be attributed to the fact 
that pumice (a highly porous volcanic rock) has a lower thermal conductivity than cement paste [34]. It has also been reported that the effective thermal conductivity of cement mortar increased with increasing free moisture content $\phi_{w}[27,28,33,35]$. Jin et al. [35] measured the thermal conductivity of autoclaved aerated concrete with increasing free moisture content at $20^{\circ} \mathrm{C}$ using a transient plane source method. The thermal conductivity was found to increase by about $13 \%$ per degree of free moisture content ranging between 0 and $15 \%$ but increased by $2 \%$ per degree of free moisture content beyond [35]. Kim et al. [33] measured the thermal conductivity of fully saturated and dry cement mortar samples containing sand and stone aggregates made with different w/c ratio and aggregate volume fraction at temperatures between $20-60^{\circ} \mathrm{C}$ using a transient hot wire method. The effective thermal conductivity of these cement mortar samples was found to increase by up to $30 \%$ as the free moisture content $\phi_{w}$ increased from 0 (fully dry) to 1 (fully saturated) [33]. It also decreased by up to $16 \%$ as the sample temperature increased from $20^{\circ} \mathrm{C}$ and $60^{\circ} \mathrm{C}$ [33]. Additionally, the authors found very little change in the thermal conductivity of these samples over time when measured over a 28-day hardening period [33]. However, the conditions of the hardening process were not described. Overall, these results were consistent with the findings of previous studies $[25,27,28,35]$ and highlight the numerous factors that can affect the thermal conductivity of cementitious composites.

Finally, adding PCM to cementitious composites has been reported to decrease the effective thermal conductivity $[8,36,37]$. Table 3 summarizes the studies reporting the effective thermal conductivity of cement mortar containing PCM either impregnated in the mortar or microencapsulated $[8,36,37]$. Both $\mathrm{Xu}$ and $\mathrm{Li}[36]$ and Zhang et al. [37] found that the thermal conductivity of cement mortar decreased linearly with increasing impregnated PCM mass fraction up to 0.3. Hunger et al. [8] reported the experimentally measured thermal conductivity of concrete containing various volume fractions of microencapsulated PCM. The authors measured the thermal conductivity of self-compacting concrete containing up to 0.12 vol.\% (5 wt.\%) of microencapsulated PCM between 19 and $28^{\circ} \mathrm{C}$ using the hot wire method. The specific heat and density of these samples were also measured independently. To the 
best of our knowledge, the thermal conductivity of other types of cementitious composites containing microencapsulated PCM has not been reported.

\subsection{Effective medium approximations}

Several effective medium approximations (EMAs) have been proposed to predict the effective thermal conductivity of three-component core-shell-matrix composites such as cement paste with embedded microencapsulated PCM [38-43]. For example, Felske [38] derived an EMA to predict the effective thermal conductivity of composites with randomly distributed monodisperse spherical microcapsules embedded in a continuous matrix. In absence of contact resistance at the shell-matrix interface, the effective thermal conductivity of three-component core-shell-matrix composites can be written as [38],

$$
k_{e f f}=\frac{2 k_{m}\left(1-\phi_{c}-\phi_{s}\right)\left(3+2 \frac{\phi_{s}}{\phi_{c}}+\frac{\phi_{s} k_{c}}{\phi_{c} k_{s}}\right)+\left(1+2 \phi_{c}+2 \phi_{s}\right)\left[\left(3+\frac{\phi_{s}}{\phi_{c}}\right) k_{c}+2 \frac{\phi_{s} k_{s}}{\phi_{c}}\right]}{\left(2+\phi_{c}+\phi_{s}\right)\left(3+2 \frac{\phi_{s}}{\phi_{c}}+\frac{\phi_{s} k_{c}}{\phi_{c} k_{s}}\right)+\left(1-\phi_{c}-\phi_{s}\right)\left[\left(3+\frac{\phi_{s}}{\phi_{c}}\right) \frac{k_{c}}{k_{m}}+2 \frac{\phi_{s} k_{s}}{\phi_{c} k_{m}}\right]} .
$$

Here, $k$ and $\phi$ are the thermal conductivity and volume fraction and the subscripts $c, s$, and $m$ refer to the core, shell, and matrix, respectively. Predictions of this model have been shown to agree very well with those obtained from detailed numerical simulations of spherical monodisperse and polydisperse core-shell-matrix particles ordered or randomly distributed in a continuous matrix. Wide ranges of constituent thermal conductivities and volume fractions were considered [44]. However, to the best of our knowledge, the Felske model [38] has not yet been validated against experimental measurements.

The present study aims to measure the effective thermal conductivity of cement paste and cement mortar without and with microencapsulated PCM for volume fraction $\phi_{c+s}$ ranging from 0 to 0.3 and temperature between 10 and $50^{\circ} \mathrm{C}$ using the guarded hot plate method. The experimentally measured effective thermal conductivity values were compared with predictions by the Felske model [38]. Once validated against experimental data, the Felske model [38] could be used in the thermal design of cementitious PCM composites for various applications. 


\section{Materials and Methods}

\subsection{Sample synthesis}

Two types of cementitious samples were investigated in this study, namely cement paste and cement mortar, each without and with microencapsulated PCM. The cementitious composite samples were prepared as per ASTM C305 [45]. Cement paste samples were made using Type I ordinary Portland cement (OPC) mixed with deionized (DI) water at a constant $\mathrm{w} / \mathrm{c}$ ratio of 0.45 . Water-reducing admixture (Glenium 7500, BASF Corporation) was added up to $2 \%$ of the cement paste mass to improve the fluidity of the mixture. The PCM microcapsules (MPCM24D, MicroTek Laboratories Inc) consisted of a polymeric melamineformaldehyde shell (10-15 mass \%) surrounding a paraffinous core (85-90 mass \%) with a melting temperature around $24^{\circ} \mathrm{C}$ [46]. The microcapsules featured a mean outer particle diameter of 17-20 $\mu \mathrm{m}[47]$ and an effective density of $900 \mathrm{~kg} / \mathrm{m}^{3}$ [46]. The PCM microcapsules volume fraction $\phi_{c+s}$ in the cement paste ranged from 0 to 0.3 in 0.1 increments. Cement mortar samples were synthesized by adding quartz sand (U.S. Silica Company) to the cement paste. The ASTM quartz sand was graded between $600 \mu \mathrm{m}$ and $150 \mu \mathrm{m}$ sieves, with 96$100 \%$ passing through the $600 \mu \mathrm{m}$ sieve and $0-4 \%$ passing through the $150 \mu \mathrm{m}$ sieve [48]. The quartz has a reported density of $2650 \mathrm{~kg} / \mathrm{m}^{3}$ [49]. The PCM microcapsule volume fraction $\phi_{c+s}$ in cement mortar ranged from 0 to 0.2 in 0.1 increments. The volume fractions of graded quartz sand $\phi_{q}$ and of PCM microcapsules $\phi_{c+s}$ in the mortar samples were such that their sum $\phi_{q}+\phi_{c+s}$ was kept constant and equal to 0.55 .

The cementitious composite samples were cast into a cylindrical acrylic mold $38 \mathrm{~mm}$ in height and $50.8 \mathrm{~mm}$ in inner diameter. The samples were cured in sealed humid conditions for 24 hours. After initial curing, the samples were removed from the mold and the top and bottom of the sample were polished manually with 600 grit sand paper. All samples were aged in ambient air at room temperature and an average relative humidity around $60 \%$ for 28 days to ensure that they had fully hardened and featured a stable microstructure $[31,32]$. Samples were synthesized in duplicates since the guarded hot plate method requires two 
identical samples to perform the thermal conductivity measurements. In order to measure $k_{e f f}$, two radial holes were drilled and two thermocouples were embedded along the vertical axis of each aged sample, separated by an axial distance $L$ of $25 \mathrm{~mm}$. Thermal cement (Arctic Alumina) with a thermal conductivity around $1 \mathrm{~W} /(\mathrm{m} \cdot \mathrm{K})$ was used to fix the thermocouples in place and achieve good thermal contact with the composite material.

\subsection{Density measurements}

The effective density $\rho_{\text {eff }}$ of the cementitious composite samples was determined by measuring their volume $V$ and their mass $m$ independently at room temperature, i.e., $\rho_{\text {eff }}=m / V$. These measurements were performed after the samples had aged for 28 days. The effective density of a few plain cement paste and mortar samples was also measured daily during the hardening and aging processes to determine the sample age beyond which the density remained constant. Moreover, once the thermal conductivity measurements were performed, the density of each sample investigated was measured for oven dry and fully saturated moisture conditions. To achieve a fully dry state, samples were dried at $105^{\circ} \mathrm{C}$ in a sealed oven for $24 \mathrm{~h}$ [27]. To achieve a fully saturated state, they were submerged in boiling DI water for $5 \mathrm{~h}$ [27]. Then, the free moisture content $\phi_{w}$ in the aged samples of effective density $\rho_{\text {eff }}$ was determined from the measured effective densities of fully dry $\rho_{d r y}$ and fully saturated $\rho_{\text {sat }}$ samples according to $[35,50]$,

$$
\phi_{w}=\frac{\rho_{e f f}-\rho_{d r y}}{\rho_{s a t}-\rho_{d r y}}
$$

\subsection{Guarded hot plate apparatus}

To measure the effective thermal conductivity of the cementitious composite specimens, a guarded hot plate apparatus was designed and fabricated in accordance with ASTM C17713 [22]. Figure 1 illustrates the entire experimental setup including (i) the guarded hot plate test section, (ii) a cooling/heating recirculating chiller (Polystat, Cole-Parmer), (iii) two flow meters (FLR-1009, Omega), (iv) two variable DC power generators (Dual DC power 
supply, Beckman Industrial), (v) two 10-channel data acquisition board (DAQ) (OMB-DAQ56, Omega), and (vi) a personal computer. The guarded hot plate method require two identical samples denoted by A and B. Each sample was secured between custom-made hot and cold plates. A vertical clamp was used to hold the test section together and to ensure good thermal contact between the samples and the hot and cold plates. The entire test section was wrapped in $5 \mathrm{~cm}$ thick microporous insulation (Cerablanket, Morgan Thermal Ceramics) with a thermal conductivity of $0.07 \mathrm{~W} /(\mathrm{m} \cdot \mathrm{K})$ and a density of $64 \mathrm{~kg} / \mathrm{m}^{3}$ to reduce heat losses [51]. The cold plates were connected to the recirculating chiller by flexible pipes directing the chiller coolant in and out of each cold plate. The volumetric flow rate $\dot{Q}_{A}$ and $\dot{Q}_{B}$ of the coolant into cold plates A and B were measured by a digital flow meter attached to each inlet pipe. Each DAQ input had a built-in cold-junction for reference measurements. DASYlab software was used to record the data.

Figure 2 shows details of the guarded hot plate test section, consisting of a hot plate and two cold plates separated by two identical samples A and B. The hot plate assembly consisted of a metered heater and of a concentric guard heater, separated by a $0.25 \mathrm{~mm}$ gap. The temperatures (i) $T_{1, A}, T_{2, A}, T_{1, B}$, and $T_{2, B}$ within samples $\mathrm{A}$ and $\mathrm{B}$, (ii) $T_{m, A}$ and $T_{m, B}$ at the bottom and top faces of the metered heater in contact with samples $\mathrm{A}$ and $\mathrm{B}$, (iii) $T_{g, A}$ and $T_{g, B}$ at the bottom and top faces of the guard heater, and (iv) $T_{c o l d, A}$ and $T_{\text {cold,B }}$ at the center of the cold plates were measured using type-T copper-constantan 24 gage thermocouples connected to the DAQ boards. Here, thermocouples measuring $T_{1, A}, T_{2, A}, T_{1, B}$, and $T_{2, B}$ were embedded directly in the sample (Figure $2 \mathrm{~b}$ ) in order to eliminate the experimental uncertainty associated with the thermal contact resistance between the sample and the hot and cold plates. The coolant temperature differences $\left(T_{w, 2, A}-T_{w, 1, A}\right)$ as well as $\left(T_{w, 2, B}-T_{w, 1, B}\right)$ between the outlet and inlet of cold plates A and B were also measured by type-T thermocouples. The inlet coolant temperature served as the thermocouple reference junction in order to reduce uncertainty. In all measurements, the temperature difference $\left(T_{w, 2, i}-T_{w, 1, i}\right)$ was very stable during the acquisition time under steady-state conditions and ranged between 
1 and $4^{\circ} \mathrm{C}$. Each thermocouple was first calibrated by immersing it in a circulating water bath held at a constant and known temperature between 10 and $40^{\circ} \mathrm{C}$ with $5^{\circ} \mathrm{C}$ increments. The thermocouple voltage was fitted as a linear function of temperature for each individual thermocouple and the fit was used in the data acquisition software.

Figure 3 shows top- and side-views of $(a, b)$ the metered heater and $(c, d)$ guard heater along with their respective dimensions. The guard section outer diameter was $50.8 \mathrm{~mm}$ and corresponded to the maximum sample diameter, as described in ASTM C177-13 [22]. Both metered and guard heaters were fabricated using copper for its large thermal conductivity ensuring uniform temperature over the hot plate surfaces. Resistance heating 22 gauge nichrome-60 wires were embedded in $0.8 \mathrm{~mm}$ deep circular grooves machined at the top of both heaters, as illustrated in Figures 3a and 3c. The wires were held in place using electrically insulating thermal cement (Arctic Alumina). Then, a $1.8 \mathrm{~mm}$ thick copper lid was attached to the top of each heater using thermal cement (Arctic Alumina) to cover the heating wires and provide a planar surface in contact with the samples. The electric resistances $R_{m}$ and $R_{g}$ of the heating wires in the metered and guard heaters were measured with a digital multimeter (34401A, Agilent) during each thermal conductivity measurement after reaching steady-state. Resistances $R_{m}$ and $R_{g}$ respectively ranged between 0.225 and $2.450 \Omega$ and between 0.614 and $6.688 \Omega$ for heater temperatures between 10 and $40^{\circ} \mathrm{C}$. Each heater was heated by Joule heating in the wire connected to a DC power supply. The voltages $U_{m}$ and $U_{g}$ across each wire were adjusted until the temperature of the metered and guard heaters were identical within $\pm 0.3^{\circ} \mathrm{C}$, per ASTM C177-13 [22]. Voltages $U_{m}$ and $U_{g}$ were also measured with a digital multimeter and ranged from 0.236 to $1.180 \mathrm{~V}$ and from 1.140 to $2.550 \mathrm{~V}$, respectively. At steady-state, the thermal energy $U_{m}^{2} / R_{m}$ dissipated in the hot plate was evenly distributed between samples A and B and ranged between 0.18 and 0.68 W.

Figure 4 shows the top- and side-views of the two identical cold plates along with their dimensions. The cold plate assembly consisted of a hollowed disc, $50.8 \mathrm{~mm}$ in diameter, made of copper with inlet and outlet copper tubes welded on diametrically opposite sides. 
Internal baffles $5.1 \mathrm{~mm}$ in depth were machined to direct the coolant around the cold plate to ensure isothermal conditions over the entire outer cold plate surface. A $1.8 \mathrm{~mm}$ thick copper disc was then welded on top to properly seal the cold plate. The inlet and outlet copper tubes had an inner and outer diameter of 5.0 and $6.4 \mathrm{~mm}$, respectively. The bottom and top cold plates, respectively in contact with samples A and B, were maintained at approximately the same temperature by manually adjusting the coolant volumetric flow rates $\dot{Q}_{A}$ and $\dot{Q}_{B}$ via individual valves. These flow rates ranged between 100 and $500 \mathrm{~mL} / \mathrm{min}$ depending on the cold bath coolant and temperature and on the desired cold plate temperature. Water or the potassium formate/water-based heat transfer fluid Dynalene HC-50 (Dynalene, Whitehall, PA) were used as the coolant for thermal conductivity measurements at cold plate temperatures above and below $10^{\circ} \mathrm{C}$, respectively.

\subsection{Data analysis}

The effective thermal conductivity of sample $i$ was calculated using Fourier's law given by

$$
k_{i}(\bar{T})=\frac{q_{i} L_{i}}{A\left(T_{2, i}-T_{1, i}\right)} \quad \text { with } i=\mathrm{A} \text { or } \mathrm{B}
$$

where $q_{i}$ is the heat transfer rate (in $\mathrm{W} / \mathrm{m}$ ) through sample $i$ and $L_{i}$ is the distance separating the thermocouples measuring $T_{1, i}$ and $T_{2, i}$. Here, $A$ is the effective surface area of the metered heater as defined by ASTM C177-13 [22] and given by $A=A_{m}+A_{\text {gap }} / 2$, where $A_{m}$ and $A_{\text {gap }}$ are the surface areas of the metered heater and of the gap section, respectively. The

thermal conductivity $k_{i}(\bar{T})$ was estimated as the average temperature $\bar{T}=\left(T_{1, i}+T_{2, i}\right) / 2$. Under steady-state conditions, the heat transfer rate $q_{i}$ was estimated as the average of the measured heat input from the guarded hot plate through each sample and of the heat removed from the cold plate $i$, i.e.,

$$
q_{i}=\frac{1}{2}\left(q_{m}+q_{w, i}\right)
$$

where $q_{m}$ and $q_{w, i}$ are the heat transfer rates measured on each side of the sample. The heat transfer rate $q_{m}$ from the guarded hot plate can be estimated from the measured voltage $U_{m}$ 
and resistance $R_{m}$ across the metered heater as $q_{m}=U_{m}^{2} / 2 R_{m}$. The heat transfer rate $q_{w, i}$ removed by the cold plate on one side of sample $i$ is expressed as $q_{w, i}=\dot{m}_{i} c_{p, w}\left(T_{w, 2, i}-T_{w, 1, i}\right)$, where $c_{p, w}$ is the specific heat of the coolant and $\left(T_{w, 2, i}-T_{w, 1, i}\right)$ is the coolant temperature difference measured between the outlet and inlet of the cold plate $i$. The mass flow rate $\dot{m}_{i}$ was determined by $\dot{m}_{i}=\rho_{w} \dot{Q}_{i}$, where $\dot{Q}_{i}$ is the volumetric flow rate measured by the digital flow meter and $\rho_{w}$ is the density of the coolant at $10^{\circ} \mathrm{C}$, taken as $999.8 \mathrm{~kg} / \mathrm{m}^{3}$ [29] and $1345 \mathrm{~kg} / \mathrm{m}^{3}$ [52] for water and Dynalene HC-50, respectively. The specific heat $c_{p, w}$ at $10^{\circ} \mathrm{C}$ was taken as $4.19 \mathrm{~kJ} /(\mathrm{kg} \cdot \mathrm{K})[29]$ and $2.68 \mathrm{~kJ} /(\mathrm{kg} \cdot \mathrm{K})$ [52] for water and Dynalene HC-50, respectively. Finally, the temperature measurements of $T_{1, i}$ and $T_{2, i}$ in samples $\mathrm{A}$ and $\mathrm{B}$ were averaged over 30 minute intervals to determine the thermal conductivities $k_{A}$ and $k_{B}$ of samples A and B. Then, the effective thermal conductivity of the cementitious composite sample was estimated as

$$
k_{e f f}(T)=\frac{1}{2}\left(\bar{k}_{A}+\bar{k}_{B}\right) \text { with } \bar{k}_{i}(\bar{T})=\frac{1}{n} \sum_{j=1}^{n} k_{i}(j)
$$

where $n$ is the total number of measurements of $k_{A}(j)$ and $k_{B}(j)$. A minimum of four consecutive measurements of $k_{A}$ and $k_{B}$ were performed, as required per ASTM C177-13 [22].

\subsection{Operation and procedure}

Measurements were performed once samples had aged for at least 28 days. First, identical samples A and B instrumented with thermocouples were placed in the test section and the clamp was tightened. The power generators connected to the resistance heating wires were then turned on and the voltages $U_{m}$ and $U_{g}$ were adjusted until the metered and guard heaters reached steady-state temperatures falling within $\pm 0.3^{\circ} \mathrm{C}$ of each other. Meanwhile,

the recirculating chiller was set to the desired cold plate temperature. The flow rates $\dot{Q}_{A}$ and $\dot{Q}_{B}$ to the cold plates were then adjusted until both cold plates A and B had temperatures within $\pm 0.3^{\circ} \mathrm{C}$ of one another. Steady-state conditions were considered to be reached when the temperatures of the hot and cold plate surfaces did not fluctuate by more than $0.3^{\circ} \mathrm{C}$ over three 30 minute intervals [22]. Then, the temperatures $T_{1, A}$ and $T_{2, A}$ in sample A 
and $T_{1, B}$ and $T_{2, B}$ in sample $B$ were recorded and averaged over 30 minute intervals [22]. A single measurement of effective thermal conductivity $\bar{k}_{A}$ and $\bar{k}_{B}$ at a given temperature lasted about 8 hours, corresponding to the time for the apparatus to reach steady-state and to record at least four consecutive measurements of $k_{A}$ and $k_{B}$.

\subsection{Experimental uncertainty}

The uncertainty in the thermal conductivity measurement of sample $i$ depended on the systematic error in the measurements of $q_{i}, A, L_{i}, T_{1, i}$, and $T_{2, i}$. Based on Equation (4), it was expressed as,

$$
\frac{\Delta k_{i}}{k_{i}}=\left[\left(\frac{\Delta q_{i}}{q_{i}}\right)^{2}+\left(\frac{\Delta A}{A}\right)^{2}+\left(\frac{\Delta L_{i}}{L_{i}}\right)^{2}+\frac{\Delta T_{2, i}^{2}+\Delta T_{1, i}^{2}}{\left(T_{2, i}-T_{1, i}\right)^{2}}\right]^{1 / 2}
$$

where $\Delta x$ is the systematic error associated with variable $x$. The experimental uncertainty associated with $q_{i}$ was due to heat losses through the samples, resistance between the sample and the hot and cold plates, and heat losses through the gap $[53,54]$. It can be written as,

$$
\frac{\Delta q_{i}}{q_{i}}=\left[\left(\frac{\Delta q_{m}}{q_{m}}\right)^{2}+\left(\frac{\Delta q_{w, i}}{q_{w, i}}\right)^{2}+2 \frac{\sigma_{q}^{2}}{q_{i}^{2}}\right]^{1 / 2} .
$$

Here, $\sigma_{q}$ denotes the standard deviation of the heat transfer rate $q_{i}$ with variance $\sigma_{q}^{2}$ estimated as,

$$
\sigma_{q}^{2}=\frac{1}{2}\left(\frac{q_{\text {loss }, i}}{2}\right)^{2}
$$

The heat loss $q_{l o s s, i}$ for sample $i$ can be estimated as the difference between the heat input $q_{m}=U_{m}^{2} / 2 R_{m}$ supplied by the hot plate to each sample and the heat transfer rate $q_{w, i}=$ $\dot{m}_{i} c_{p, w}\left(T_{w, 2, i}-T_{w, 1, i}\right)$ carried by the coolant passing through the cold plate, i.e.,

$$
q_{l o s s, i}=\frac{1}{2} \frac{U_{m}^{2}}{R_{m}}-\dot{m}_{i} c_{p, w}\left(T_{w, 2, i}-T_{w, 1, i}\right)
$$

The uncertainties associated with $q_{m}$ and $q_{w, i}$ are respectively expressed as,

$$
\left(\frac{\Delta q_{m}}{q_{m}}\right)^{2}=\left(2 \frac{\Delta U_{m}}{U_{m}}\right)^{2}+\left(\frac{\Delta R_{m}}{R_{m}}\right)^{2}
$$


and

$$
\left(\frac{\Delta q_{w, i}}{q_{w, i}}\right)^{2}=\left(\frac{\Delta \dot{Q}_{i}}{\dot{Q}_{i}}\right)^{2}+\left(\frac{\Delta \rho_{w}}{\rho_{w}}\right)^{2}+\left(\frac{\Delta c_{p, w}}{c_{p, w}}\right)^{2}+\left(\frac{\Delta\left(T_{w, 2, i}-T_{w, 1, i}\right)}{T_{w, 2, i}-T_{w, 1, i}}\right)^{2} .
$$

The uncertainty in voltage $\Delta U_{m}$ and resistance $\Delta R_{m}$ correspond to the measurement uncertainty from the digital multimeter and were on the order of $\pm 0.001 \mathrm{~V}$ and $\pm 0.001 \Omega$. The uncertainty in the coolant volumetric flow rate $\Delta \dot{Q}_{i}$ was on the order of $\pm 1 \mathrm{~mL} / \mathrm{min}$ based on the manufacturer calibration. The coolant density $\rho_{w}$ and specific heat $c_{p, w}$ were taken as constant values, they had uncertainties $\Delta \rho_{w}$ of $10 \mathrm{~kg} / \mathrm{m}^{3}$ and $\Delta c_{p, w}$ of $0.05 \mathrm{~kJ} /(\mathrm{kg} \cdot \mathrm{K})$ due to fluctuations in the coolant temperature. The uncertainty in the axial distance between the two thermocouples in each sample $\Delta L_{i}$ was on the order of $\pm 0.5 \mathrm{~mm}$. Thermal expansion was deemed to be negligible given the small difference between the temperatures at which thermal conductivity and the distance $L_{i}$ were measured $[55,56]$. The uncertainty $\Delta A$ was estimated as $\Delta A=\Delta A_{m}+1 / 2 \Delta A_{g a p}$, where uncertainties $\Delta A_{m}$ and $\Delta A_{\text {gap }}$ in the surface area measurements were on the order of $\pm 0.5 \mathrm{~mm}^{2}$. On completion of the thermocouple calibration procedure, the uncertainty in temperature measurements $\Delta T_{2, i}, \Delta T_{1, i}$, and $\Delta\left(T_{w, 2, i}-T_{w, 1, i}\right)$ were on the order of $\pm 0.5^{\circ} \mathrm{C}$. The heat loss $q_{\text {loss }, i}$, given by Equation (10), was the main contribution to the experimental uncertainty $\Delta q_{i} / q_{i}$ and represented 10-20\% of the averaged heat input $q_{m}$. The uncertainty terms for the sample and coolant temperature measurements and the heat loss were also major sources of error. Thus, the test section was carefully insulated and large temperature differences $\left(T_{2, i}-T_{1, i}\right)$ across the sample and $\left(T_{w, 2, i}-T_{w, 1, i}\right)$ across the cold plate were imposed to reduce uncertainty.

Finally, uncertainty in the measured effective thermal conductivity $k_{\text {eff }}$ of the cementitious composite materials of samples A and B was expressed as

$$
\frac{\Delta k_{e f f}}{k_{e f f}}=\sum_{j=1}^{n} \frac{1}{2 n}\left[\Delta k_{A}(j)^{2}+\Delta k_{B}(j)^{2}+2 \operatorname{cov}\left(k_{A}(j), k_{B}(j)\right)\right]^{1 / 2}
$$

where $\Delta k_{A}$ and $\Delta k_{B}$ are determined from Equations (7) to (12) while $\operatorname{cov}\left(k_{A}(j), k_{B}(j)\right)$ is the covariance of $k_{A}$ and $k_{B}$ defined as,

$$
\operatorname{cov}\left(k_{A}, k_{B}\right)=\frac{\left(k_{A}(j)-\bar{k}_{A}\right)\left(k_{B}(j)-\bar{k}_{B}\right)}{n} .
$$


This covariance term was used to account for the deviation of the different measurements of $k_{A}$ and $k_{B}$ from their respective mean $\bar{k}_{A}$ and $\bar{k}_{B}$, respectively. In practice, measurements of $k_{A}(j)$ and $k_{B}(j)$ were highly repeatable. Intersample variability, accounted for by $\operatorname{cov}\left(k_{A}, k_{B}\right)$, led to an average difference between $k_{A}(j)$ and $k_{B}(j)$ of $0.15 \mathrm{~W} /(\mathrm{m} \cdot \mathrm{K})$. Overall, the experimental uncertainty in $k_{\text {eff }}$ was mainly due to uncertainties in temperature measurements $\Delta T_{1, i}, \Delta T_{2, i}$, and $\Delta\left(T_{w, 2, i}-T_{w, 1, i}\right)$.

\subsection{Validation}

In order to validate the guarded hot plate apparatus, the experimental methodology, and the data analysis described in the previous sections, the thermal conductivity of two certified reference material (CRM) Pyrex 7740 glass cylindrical samples (Corning 7740, Esco Optics) was measured. The samples were $38 \mathrm{~mm}$ in thickness and $50.8 \mathrm{~mm}$ in diameter. Their thermal conductivity $k_{p}$ (in $\mathrm{W} /(\mathrm{m} \cdot \mathrm{K})$ ) was known as a function of temperature, with $\pm 6.5 \%$ uncertainty, between -75 and $195^{\circ} \mathrm{C}$ and expressed as [57],

$$
k_{p}(T)=1.1036+1.659 \times 10^{-3} T-3.982 \times 10^{-5} T^{2}+6.764 \times 10^{-9} T^{3}
$$

where $\mathrm{T}$ is the sample temperature $\left(\right.$ in $\left.{ }^{\circ} \mathrm{C}\right)$.

Figure 5 compares the experimentally measured thermal conductivity of Pyrex glass with predictions by Equation (15) for temperature ranging from 10 to $40^{\circ} \mathrm{C}$. The error bars illustrate the systematic and random error associated with each measurement estimated by Equations (7) to (14). It is evident that the experimentally measurements agreed, within experimental uncertainty, with predictions by Equation (15) over the entire temperature range considered. These results established that the guarded hot plate apparatus, experimental methodology, and the data analysis were valid and could be used to accurately measure the thermal conductivity of cementitious composite samples. 


\section{Results and Discussion}

\subsection{Sample density and free moisture content}

Figure 6 shows the measured density of two cement mortar samples with volume fraction $\phi_{q}$ of graded quartz sand of 0.35 and 0.45 but without microencapsulated PCM, as a function of time over a 28 day hardening period. It demonstrates that density decreased significantly during the first 4 days and remained constant after 20 days.

Table 4 summarizes (i) the effective density $\rho_{\text {eff }}$, (ii) the dry $\rho_{d r y}$, and (iii) saturated $\rho_{\text {sat }}$ effective densities, as well as (iv) the free moisture content $\phi_{w}$ measured for each samples aged more than 28 days. It indicates that the free moisture content of the cement paste-PCM composite samples was around 0.2 - 0.4 while that of the cement mortar-PCM composite samples was less than 0.1. This difference could be attributed to the fact that the graded quartz sand does not absorb water, unlike porous cement paste.

\subsection{Cement samples with microencapsulated PCM}

Figure 7 a plots the effective thermal conductivity $k_{\text {eff }}(T)$ of aged cement paste containing microencapsulated PCM with volume fraction $\phi_{c+s}$ of $0,0.1,0.2$, and 0.3 as a function of temperature $T$ between 10 and $50^{\circ} \mathrm{C}$. The error bars correspond to the systematic and random error associated with each measurement, as estimated by Equations (7) to (14). It indicates that the effective thermal conductivity $k_{\text {eff }}(T)$ of each specimen was nearly independent of temperature between 10 and $50^{\circ} \mathrm{C}$. Note that fluctuations in $k_{e f f}(T)$ were due to experimental uncertainty and were not statistically significant. In addition, this temperature range encompassed the entire phase change temperature window of the PCM [47]. This indicates that the effective thermal conductivity of cement paste-PCM composites was not affected appreciably by the occurrence of phase change, unlike their effective specific heat [47]. This can be attributed to the fact that the solid and liquid phases of organic PCMs have been reported to have similar thermal conductivities $[58,59]$. Note also that the temperature range considered was relatively 


\section{narrow $\left(10-50^{\circ} \mathrm{C}\right)$ although representative of actual practical conditions.}

Figure $7 \mathrm{~b}$ plots the temperature-averaged effective thermal conductivity $\left\langle k_{\text {eff }}\right\rangle$ of cement paste-PCM composite specimens as a function of microencapsulated PCM volume fraction $\phi_{c+s}$ ranging from 0 to 0.3 . It indicates that the average effective thermal conductivity $<k_{e f f}>$ decreased almost linearly as the microencapsulated PCM volume fraction $\phi_{c+s}$ increased. This can be attributed to the fact that the thermal conductivity of both the PCM core and shell was smaller than that of cement paste. Figure $7 \mathrm{~b}$ also shows predictions by the Felske model [38], given by Equation (2), using the effective thermal conductivity of plain cement paste (i.e., $\left.\phi_{c+s}=0\right)$ measured as $1.14 \pm 0.17 \mathrm{~W} /(\mathrm{m} \cdot \mathrm{K})$ as the matrix thermal conductivity $<k_{m}>$. Here, the thermal conductivity of the MPCM24D core $k_{c}$ was taken as $0.21 \mathrm{~W} /(\mathrm{m} \cdot \mathrm{K})[46,60]$ and that of the shell $k_{c}$ as $0.42 \mathrm{~W} /(\mathrm{m} \cdot \mathrm{K})$ near room temperature [61]. Figure $7 \mathrm{~b}$ indicates that predictions by the Felske model [38] agreed well with experimental measurements. The relative difference was less than $4.5 \%$. Note that predictions by the three-phase EMAs proposed by Brailsford and Major [40] were also in good agreement with experimental data. Finally, Table 4 summarizes the effective thermal conductivity $<k_{e f f}>$ of cement paste-PCM composites for each composition averaged over the temperature ranged considered.

\subsection{Cementitious mortar samples with microencapsulated PCM}

Figure 8a plots the effective thermal conductivity $k_{\text {eff }}(T)$ of cement mortar containing graded quartz sand with volume fraction $\phi_{q}$ and microencapsulated PCM with volume fraction $\phi_{c+s}$ such that $\phi_{q}+\phi_{c+s}=0.55$. Here also, all samples were aged more than 28 days in ambient air. Figure 8 indicates that the effective thermal conductivity of each cement mortar composite specimen was nearly independent of temperature between 10 and $40^{\circ} \mathrm{C}$. Here also, it was not affected appreciably by the occurrence of phase change. Figure 8a shows that for constant volume fraction $\phi_{q}+\phi_{c+s}=0.55$, the effective thermal conductivity of cement mortarPCM composites decreased with increasing PCM volume fraction. Table 4 lists the effective thermal conductivity $\left\langle k_{e f f}\right\rangle$ averaged over the temperature ranged considered for each 
composition with various values of $\phi_{q}$ and $\phi_{c+s}$.

Figure $8 \mathrm{~b}$ plots the ratio $\left\langle k_{e f f}>/<k_{m}>\right.$ of the temperature-averaged effective thermal conductivity of cement mortar-PCM composite specimens $<k_{e f f}>$ and the cement mortar $<k_{m}>$ as a function of microencapsulated $\mathrm{PCM}$ volume fraction $\phi_{c+s}$ ranging from 0 to 0.2 . It also shows, in inset, the temperature-averaged thermal conductivity $<k_{m}>$ of cement mortar without PCM as a function of quartz sand volume fraction $\phi_{q}$. Recall that the volume fraction of the graded quartz sand $\phi_{q}$ decreased as microencapsulated PCM was added to maintain the total aggregate volume fraction $\phi_{q}+\phi_{c+s}=0.55$. Thus, the effective matrix thermal conductivity $<k_{m}>$ changed as microencapsulated PCM was added. The measured thermal conductivity $<k_{m}>$ of cement mortar samples without PCM (i.e., $\phi_{c+s}=0$ ) and with a graded quartz volume fraction $\phi_{q}$ of $0.55,0.45$, and 0.35 was used as the matrix thermal conductivity $<k_{m}>$ for samples containing $\phi_{c+s}$ of $0,0.1$, and 0.2 , respectively. Figure 8a indicates that the thermal conductivity ratio $\left\langle k_{e f f}\right\rangle /\left\langle k_{m}>\right.$ decreased as the microencapsulated PCM volume fraction $\phi_{c+s}$ increased and as the graded quartz sand volume fraction $\phi_{q}$ simultaneously decreased. This can be attributed to the fact that the thermal conductivities of the core and shell of the PCM microcapsules were smaller than that of cement mortar and quartz. Figure 8b also shows predictions by the Felske model [38], given by Equation (2). Here also, predictions by the Felske model [38] for cement mortar-PCM composites agreed very well with experimental measurements.

\section{Conclusion}

A guarded hot plate apparatus was designed, assembled, and validated to measure the effective thermal conductivity of (i) OPC paste and (ii) cement mortar containing microencapsulated PCM with volume fraction $\phi_{c+s}$ of up to 0.3 for temperatures between 10 and $40^{\circ} \mathrm{C}$. Cement paste and cement mortar samples were prepared with a constant water to cement ratio of 0.45 and aged for at least 28 days in ambient air. The cement mortar samples also included volume fraction $\phi_{q}$ of graded quartz sand such that $\phi_{q}+\phi_{c+s}=0.55$. The effective 
density $\rho_{\text {eff }}$ and free moisture content $\phi_{w}$ of all aged samples were systematically measured. For both composite types, thermal conductivity remained nearly constant between $10-50^{\circ} \mathrm{C}$ and decreased as the microencapsulated PCM volume fraction increased. It was larger for composites made of cement mortar $(1.2-1.8 \mathrm{~W} /(\mathrm{m} \cdot \mathrm{K}))$ than of OPC paste $(0.8$ $1.2 \mathrm{~W} /(\mathrm{m} \cdot \mathrm{K}))$ with the same PCM volume fraction due to the presence of quartz grains. Predictions of the effective medium approximation proposed by Felske [38] for the effective thermal conductivity of core-shell-matrix composites agreed very well with experimental measurements. These results will be instrumental in designing cementitious composite materials for energy efficient buildings and crack-resistant pavements.

\section{Acknowledgements}

This manuscript was prepared as a result of work sponsored by the California Energy Commission (Contract: PIR:-12-032), the National Science Foundation (CMMI: 1130028), an Infravation ERA-NET Plus grant (31109806.0001), and the University of California, Los Angeles (UCLA). It does not necessarily represent the views of the Energy Commission, its employees, the State of California, or the National Science Foundation. The Energy Commission, the State of California, its employees, contractors, and subcontractors make no warranty, express or implied, and assume no legal liability for the information in this document; nor does any party represent that the use of this information will not infringe upon privately owned rights. This manuscript has not been approved or disapproved by the California Energy Commission nor has the California Energy Commission passed upon the accuracy or adequacy of the information in this paper.

\section{References}

[1] F. Kuznik, D. David, K. Johannes, and J.-J. Roux, "A review on phase change materials integrated in building walls", Renewable and Sustainable Energy Reviews, vol. 15, no. 
1, pp. 379-391, 2011.

[2] T.-C. Ling and C.-S. Poon, "Use of phase change materials for thermal energy storage in concrete: An overview", Construction and Building Materials, vol. 46, pp. 55-62, 2013.

[3] M.K. Rathod and J. Banerjee, "Thermal stability of phase change materials used in latent heat energy storage systems: a review", Renewable and Sustainable Energy Reviews, vol. 18, pp. 246-258, 2013.

[4] F. Fernandes, S. Manari, M. Aguayo, K. Santos, T. Oey, Z. Wei, G. Falzone, N. Neithalath, and G. Sant, "On the feasibility of using phase change materials (PCMs) to mitigate thermal cracking in cementitious materials", Cement and Concrete Composites, vol. 51, pp. 14-26, 2014.

[5] D.P. Bentz and R. Turpin, "Potential applications of phase change materials in concrete technology", Cement and Concrete Composites, vol. 29, no. 7, pp. 527-532, 2007.

[6] L.F. Cabeza, C. Castellon, M. Nogues, M. Medrano, R. Leppers, and O. Zubillaga, "Use of microencapsulated PCM in concrete walls for energy savings", Energy and Buildings, vol. 39, no. 2, pp. 113-119, 2007.

[7] D. Zhou, C.-Y. Zhao, and Y. Tian, "Review on thermal energy storage with phase change materials (PCMs) in building applications", Applied Energy, vol. 92, pp. 593$605,2012$.

[8] M. Hunger, A.G. Entrop, I. Mandilaras, H.J.H. Brouwers, and M. Founti, "The behavior of self-compacting concrete containing micro-encapsulated phase change materials", Cement and Concrete Composites, vol. 31, no. 10, pp. 731-743, 2009.

[9] A. Sharma, V.V. Tyagi, C.R. Chen, and D. Buddhi, "Review on thermal energy storage with phase change materials and applications", Renewable and Sustainable Energy Reviews, vol. 13, no. 2, pp. 318-345, 2009. 
[10] A.M. Thiele, G. Sant, and L. Pilon, "Diurnal thermal analysis of microencapsulated PCM-concrete composite walls", Energy Conversion and Management, vol. 93, pp. 215-227, 2015.

[11] A.M. Thiele, A. Jamet, G. Sant, and L. Pilon, "Annual energy analysis of concrete containing phase change materials for building envelopes", Energy Conversion and Management, vol. 103, pp. 374-386, 2015.

[12] V.V. Tyagi, S.C. Kaushik, S.K. Tyagi, and T. Akiyama, "Development of phase change materials based microencapsulated technology for buildings: a review", Renewable and Sustainable Energy Reviews, vol. 15, no. 2, pp. 1373-1391, 2011.

[13] T. Log and S.E. Gustafsson, "Transient plane source (TPS) technique for measuring thermal transport properties of building materials", Fire and Materials, vol. 19, no. 1, pp. 43-49, 1995.

[14] S.E. Gustafsson, "Transient hot strip techniques for measuring thermal conductivity and thermal diffusivity", The Rigaku Journal, vol. 4, no. 1/2, pp. 16-28, 1987.

[15] J.J. Healy, J.J. De Groot, and J. Kestin, "The theory of the transient hot-wire method for measuring thermal conductivity", Physica B $\mathscr{G}$ C, vol. 82, no. 2, pp. 392-408, 1976.

[16] U. Hammerschmidt and V. Meier, "New transient hot-bridge sensor to measure thermal conductivity, thermal diffusivity, and volumetric specific heat", International Journal of Thermophysics, vol. 27, no. 3, pp. 840-865, 2006.

[17] W.J. Parker, R.J. Jenkins, C.P. Butler, and G.L. Abbott, "Flash method of determining thermal diffusivity, heat capacity, and thermal conductivity", Journal of Applied Physics, vol. 32, no. 9, pp. 1679-1684, 1961.

[18] D. Kuvandykova and R. St-Laurent, "Application of the modified transient plane source technique in testing the thermal conductivity of concrete", C-Therm Technol, vol. 18, pp. 1-7, 2010. 
[19] P.S. Gaal, M.-A. Thermitus, and D.E. Stroe, "Thermal conductivity measurements using the flash method", Journal of Thermal Analysis and Calorimetry, vol. 78, no. 1, pp. 185-189, 2004.

[20] C. Bankvall, "Guarded hot plate apparatus for the investigation of thermal insulations", Matériaux et Construction, vol. 6, no. 1, pp. 39-47, 1973.

[21] D. Salmon, "Thermal conductivity of insulations using guarded hot plates, including recent developments and sources of reference materials", Measurement Science and Technology, vol. 12, no. 12, pp. R89, 2001.

[22] ASTM Standard, "C177-13", Standard test method for steady-state heat flux measurements and thermal transmission properties by means of the guarded-hot plate apparatus. ASTM International, West Conshohocken, PA, USA, 2014.

[23] D.P. Bentz, "Transient plane source measurements of the thermal properties of hydrating cement pastes", Materials and Structures, vol. 40, no. 10, pp. 1073-1080, 2007.

[24] Y. Xu and D.D.L. Chung, "Increasing the specific heat of cement paste by admixture surface treatments", Cement and Concrete Research, vol. 29, no. 7, pp. 1117-1121, 1999.

[25] Y. Xu and D.D.L. Chung, "Effect of sand addition on the specific heat and thermal conductivity of cement", Cement and Concrete Research, vol. 30, no. 1, pp. 59-61, 2000.

[26] R. Demirboğa, "Thermal conductivity and compressive strength of concrete incorporation with mineral admixtures", Building and Environment, vol. 42, no. 7, pp. 2467-2471, 2007.

[27] M.I. Khan, "Factors affecting the thermal properties of concrete and applicability of its prediction models", Building and Environment, vol. 37, no. 6, pp. 607-614, 2002.

[28] D. Campbell-Allen and C.P. Thorne, "The thermal conductivity of concrete", Magazine of Concrete Research, vol. 15, no. 43, pp. 39-48, 1963. 
[29] T.L. Bergman, A.S. Lavine, F.P. Incropera, and D.P. DeWitt, Fundamentals of Heat and Mass Transfer, John Wiley \& Sons, New York City, NY, $7^{\text {th }}$ edition, 2011.

[30] K. Liu, Z. Wang, C. Jin, F. Wang, and X. Lu, "An experimental study on thermal conductivity of iron ore sand cement mortar", Construction and Building Materials, vol. 101, pp. 932-941, 2015.

[31] T.D. Brown and M.Y. Javaid, "The thermal conductivity of fresh concrete", Matériaux et Construction, vol. 3, no. 6, pp. 411-416, 1970.

[32] G. De Schutter and L. Taerwe, "Specific heat and thermal diffusivity of hardening concrete", Magazine of Concrete Research, vol. 47, no. 172, pp. 203-208, 1995.

[33] K.H. Kim, S.E. Jeon, J.K. Kim, and S. Yang, "An experimental study on thermal conductivity of concrete", Cement and Concrete Research, vol. 33, no. 3, pp. 363-371, 2003.

[34] H. Uysal, R. Demirboğa, R. Şahin, and R. Gül, "The effects of different cement dosages, slumps, and pumice aggregate ratios on the thermal conductivity and density of concrete", Cement and Concrete Research, vol. 34, no. 5, pp. 845-848, 2004.

[35] H.-Q. Jin, X.-L. Yao, L.-W. Fan, X. Xu, and Z.-T. Yu, "Experimental determination and fractal modeling of the effective thermal conductivity of autoclaved aerated concrete: Effects of moisture content", International Journal of Heat and Mass Transfer, vol. 92, pp. 589-602, 2016.

[36] B. Xu and Z. Li, "Paraffin/diatomite composite phase change material incorporated cement-based composite for thermal energy storage", Applied Energy, vol. 105, pp. 229-237, 2013.

[37] Z. Zhang, G. Shi, S. Wang, X. Fang, and X. Liu, "Thermal energy storage cement mortar containing n-octadecane/expanded graphite composite phase change material", Renewable Energy, vol. 50, pp. 670-675, 2013. 
[38] J.D. Felske, "Effective thermal conductivity of composite spheres in a continuous medium with contact resistance", International Journal of Heat and Mass Transfer, vol. 47, no. 14, pp. 3453-3461, 2004.

[39] Y.K. Park, J.G. Kim, and J.K. Lee, "Prediction of thermal conductivity of composites with spherical microballoons", Materials Transactions, vol. 49, no. 12, pp. 2781-2785, 2008.

[40] A.D. Brailsford and K.G. Major, "The thermal conductivity of aggregates of several phases, including porous materials", British Journal of Applied Physics, vol. 15, no. 3, pp. 313, 1964.

[41] M. Porfiri, N.Q. Nguyen, and N. Gupta, "Thermal conductivity of multiphase particulate composite materials", Journal of Materials Science, vol. 44, no. 6, pp. 1540-1550, 2009.

[42] W.M.J.H. Woodside and J.H. Messmer, "Thermal conductivity of porous media. I. Unconsolidated sands", Journal of Applied Physics, vol. 32, no. 9, pp. 1688-1699, 1961.

[43] T. Zakri, J.P. Laurent, and M. Vauclin, "Theoretical evidence for Lichtenecker's mixture formulae based on the effective medium theory", Journal of Physics D: Applied Physics, vol. 31, no. 13, pp. 1589, 1998.

[44] A.M. Thiele, A. Kumar, G. Sant, and L. Pilon, "Effective thermal conductivity of three-component composites containing spherical capsules", International Journal of Heat and Mass Transfer, vol. 73, pp. 177-185, 2014.

[45] ASTM Standard, "C305", Standard practice for mechanical mixing of hydraulic cement pastes and mortars of plastic consistency. ASTM International, West Conshohocken, PA, USA, 2014.

[46] "MPCM Technical Information", Tech. Rep., Microtek Laboratories Inc., Dayton, OH. 
[47] A.M. Thiele, Z. Wei, G. Falzone, B. Young, N. Niethalath, G. Sant, and L. Pilon, "Figure of merit for the thermal performance of cementitious composites containing phase change materials", Cement and Concrete Composites, vol. 65, no. 1, pp. 214-226, 2016.

[48] ASTM Standard, "C788-06", Standard specification for standard sand. ASTM International, West Conshohocken, PA, USA, 2006.

[49] T.L. Youd, "Factors controlling maximum and minimum densities of sands", ASTM Special Technical Publications, vol. 523, pp. 98-112, 1973.

[50] Z. Lafhaj, M. Goueygou, A. Djerbi, and M. Kaczmarek, "Correlation between porosity, permeability and ultrasonic parameters of mortar with variable water/cement ratio and water content", Cement and Concrete Research, vol. 36, no. 4, pp. 625-633, 2006.

[51] "Cerablanket, Cerachem, Cerachrome Blanket datasheet", Tech. Rep., Morgan Thermal Ceramics, Inc., Windsor, Berkshire, United Kingdom.

[52] "Dynalene HC Series", Tech. Rep., Dynalene Inc., Whitehall, PA.

[53] U. Hammerschmidt, "Guarded hot-plate (GHP) method: Uncertainty assessment", International Journal of Thermophysics, vol. 23, no. 6, pp. 1551-1570, 2002.

[54] H. Simmler, "Uncertainty assessment of guarded hot plate apparatus", Tech. Rep., EMPA Building Technologies Lab, Düebendorf, Switzerland, June 2008.

[55] S.L. Meyers, "Thermal expansion characteristics of hardened cement paste and of concrete", in Highway Research Board Proceedings, 1951, vol. 30, pp. 193-203.

[56] E.J. Sellevold and Ø. Bjøntegaard, "Coefficient of thermal expansion of cement paste and concrete: Mechanisms of moisture interaction", Materials and Structures, vol. 39, no. 9, pp. 809-815, 2006. 
[57] BCR Certified reference material, "BCR-039", Certified reference material BCR-039, Pyrex Glass. Institue for Reference Materials and Measurements, European Union, 2007.

[58] J.J. Eftekhar, A.A. Haji-Sheikh, and D.S. Lou, "Heat transfer enhancement in a paraffin wax thermal storage system", ASME Journal of Solar Energy Engineering, vol. 106, no. 3, pp. 299-306, 1984.

[59] A. Himran, A. Suwono, and G.A. Mansoori, "Characterization of alkanes and paraffin waxes for application as phase change energy storage medium", Energy Sources, vol. 16, no. 1, pp. 117-128, 1994.

[60] N. Ukrainczyk, S. Kurajica, and J. Šipušić, "Thermophysical comparison of five commercial paraffin waxes as latent heat storage materials", Chemical and Biochemical Engineering Quarterly, vol. 24, no. 2, pp. 129-137, 2010.

[61] R.K. Rajput, Engineering Materials 8 Metallurgy, S. Chand Publishing Limited, New Delhi, India, 1st edition, 2006. 


\section{List of Figures}

1 Experimental setup used to measure the effective thermal conductivity of cementitious PCM composites based on the guarded hot plate method. . . . 33

2 (a) Exploded cross-sectional view of the guarded hot plate test section labeling each component and (b) collapsed cross-sectional view detailing thermocouple locations and relevant notations. . . . . . . . . . . . . . .

3 Side and top-views of the metered (a) and (b) and guard (c) and (d) sections, respectively, along with their associated dimensions (all in $\mathrm{mm}$ ). . . . . . . .

4 Side (a) and top (b) views of the cold plate with its associated dimensions (all in $\mathrm{mm}) \ldots \ldots \ldots \ldots \ldots \ldots \ldots \ldots$

5 Measured thermal conductivity of Pyrex glass and the reported thermal conductivity of the Pyrex certified reference material BCR-039 given by Equation (15) [57]

6 Effective density $r h o_{e f f}$ of cement mortar containing graded quartz sand with volume fraction $\phi_{q}$ of 0.45 and 0.35 but no microencapsulated PCM $\left(\phi_{c+s}=0\right)$ over a 28 day hardening period. . . . . . . . . . . . . . . 38

7 (a) Measured effective thermal conductivity $k_{\text {eff }}$ of cement paste with a constant w/c ratio of 0.45 and volume fraction of microencapsulated PCM $\phi_{c+s}$ between 0 and 0.3 as a function of temperature $T$. (b) Temperature-averaged effective thermal conductivity $<k_{e f f}>$ as a function of $\phi_{c+s}$ with the predictions of the Felske model [38] given by Equation (2). . . . . . . . . . . . 
8 (a) Measured effective thermal conductivity of cement mortar with a constant w/c of 0.45 with volume fraction of microencapsulated PCM $\phi_{c+s}$ between 0 and 0.3 and graded quartz sand $\phi_{q}$ between 0.35 and 0.55 such that $\phi_{q}+\phi_{c+s}=0.55$ as a functions of temperature $T$. (b) Ratio of measured temperature-averaged thermal conductivity $\left\langle k_{e f f}>/<k_{m}>\right.$ of cement mortar with constant w/c of 0.45 as a function of volume fraction of microencapsulated PCM $\phi_{c+s}$ between 0 and 0.3 for graded quartz sand volume fraction $\phi_{q}$ such that $\phi_{q}+\phi_{c+s}=0.55$. Here, $<k_{m}\left(\phi_{q}\right)>$ is shown in the inset. . . . . .

\section{List of Tables}

1 Thermal conductivity measurements reported in the literature for cement paste $[23-26,33]$.

2 Thermal conductivity measurements reported in the literature for cement mor$\operatorname{tar}[25,28,33] \ldots \ldots \ldots \ldots \ldots \ldots \ldots$

3 Thermal conductivity measurements reported in the literature for cement mortar with impregnated or microencapsulated PCM $[36,37] \ldots$. . . . . . . . .

4 Temperature-averaged effective thermal conductivity $<k_{e f f}>$ of cement paste and cement mortar samples for microencapsualted PCM volume fraction $\phi_{c+s}$ ranging from 0 to 0.3 . Cement mortar samples had a constant volume fraction of graded quartz sand and microencapsulated PCM such that $\phi_{q}+\phi_{c+s}=0.55$. 44 


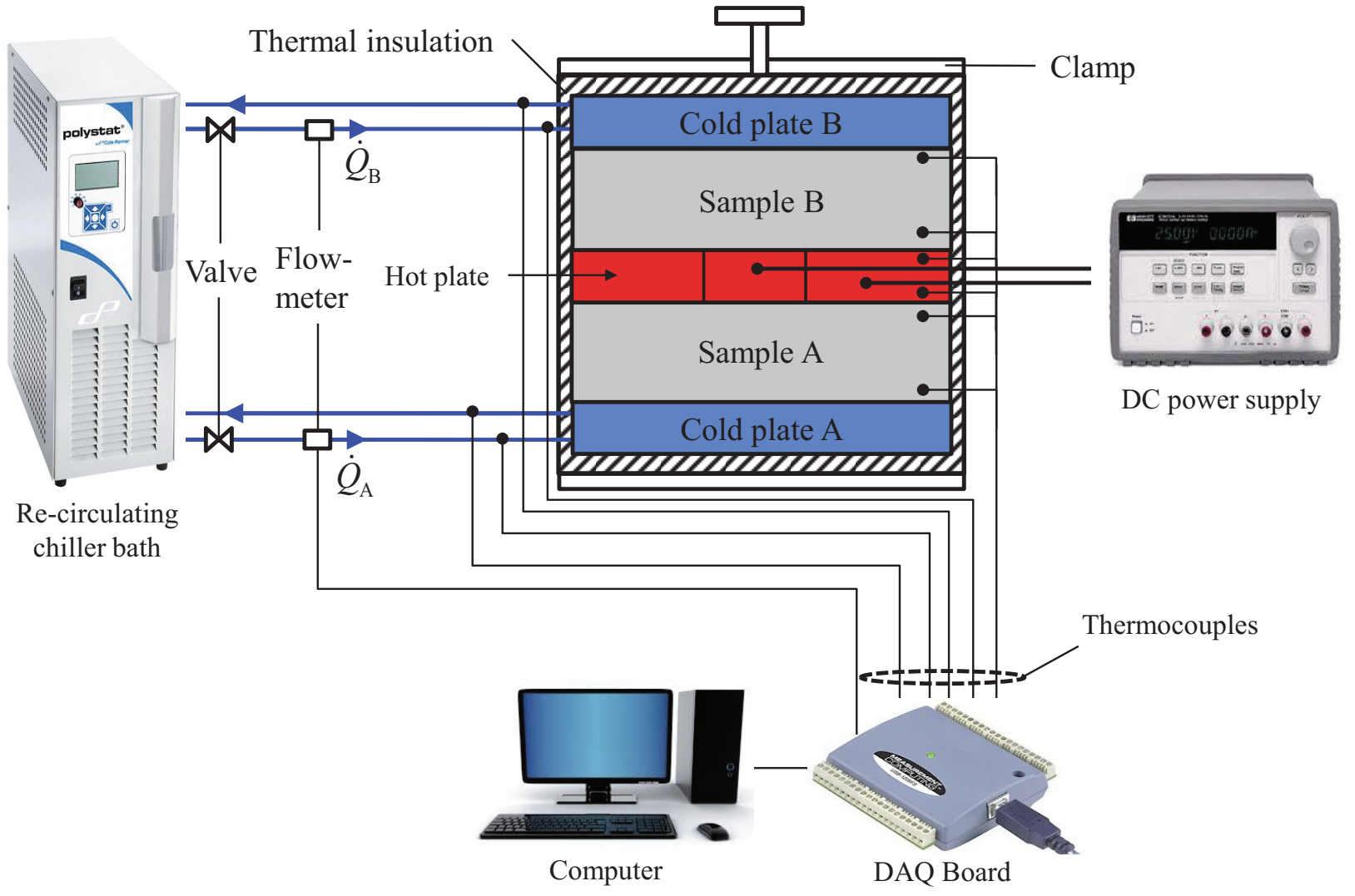

Figure 1: Experimental setup used to measure the effective thermal conductivity of cementitious PCM composites based on the guarded hot plate method. 

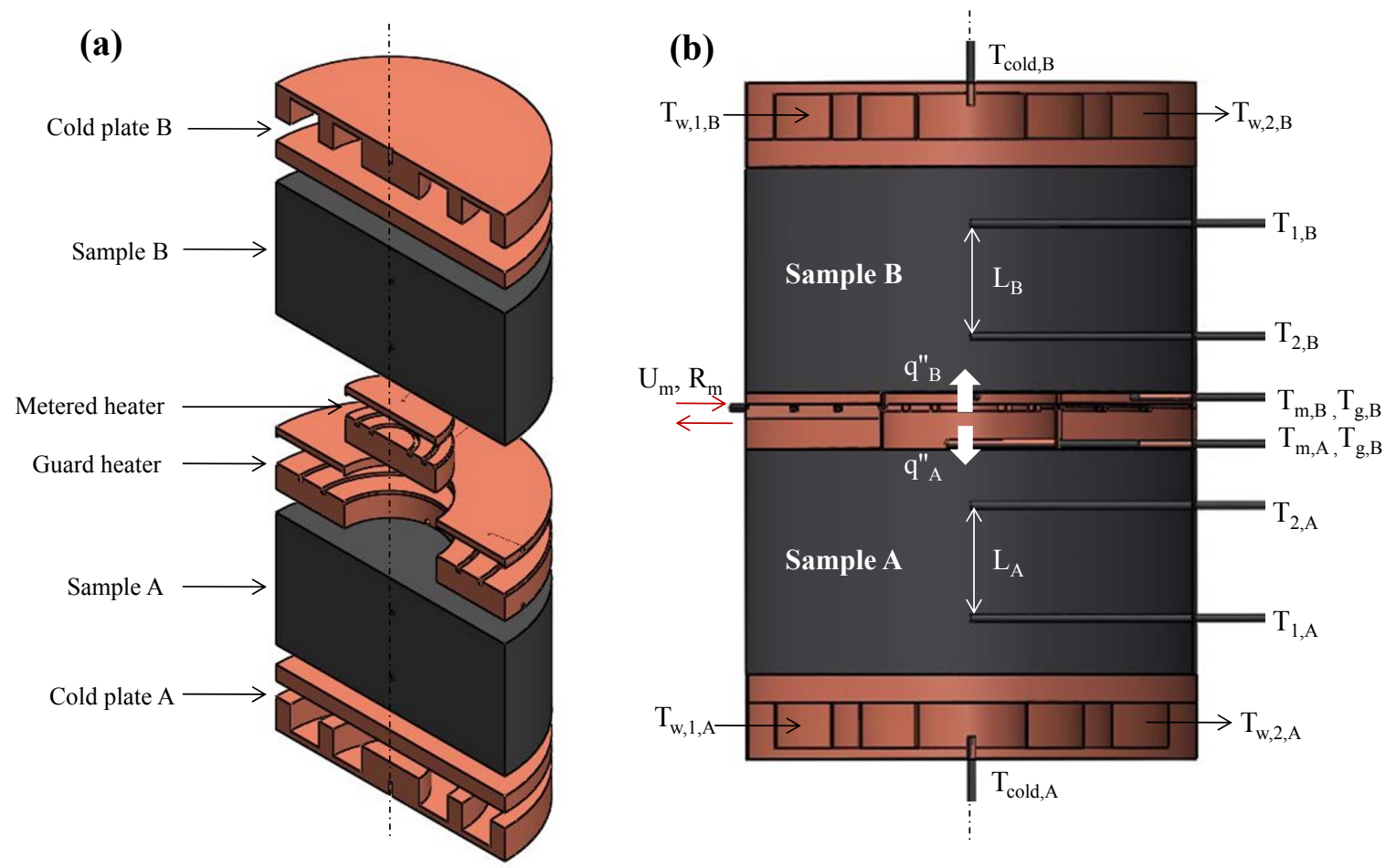

Figure 2: (a) Exploded cross-sectional view of the guarded hot plate test section labeling each component and (b) collapsed cross-sectional view detailing thermocouple locations and relevant notations. 
(a)

(c)
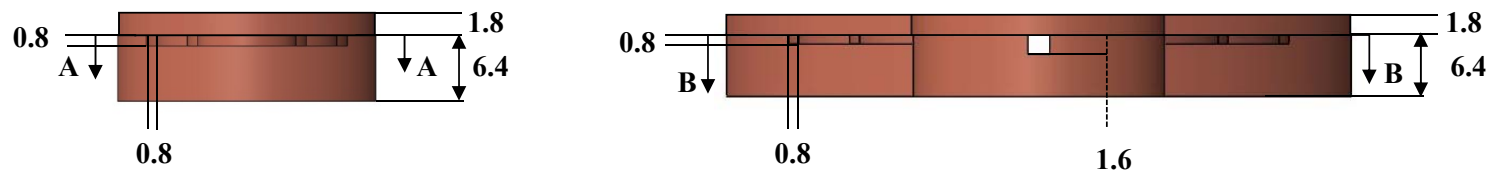

(b)

View A-A

(d)

View B-B
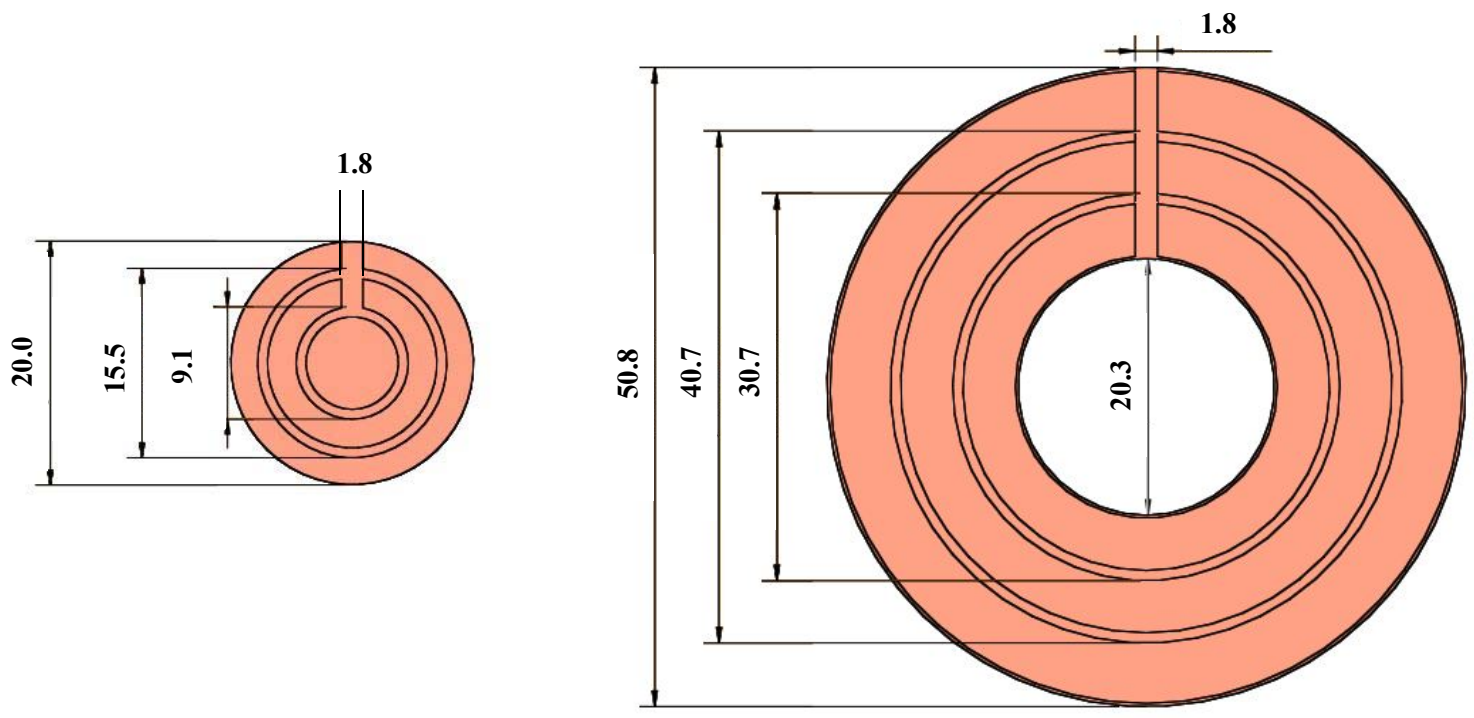

Figure 3: Side and top-views of the metered (a) and (b) and guard (c) and (d) sections, respectively, along with their associated dimensions (all in $\mathrm{mm}$ ). 
(a)

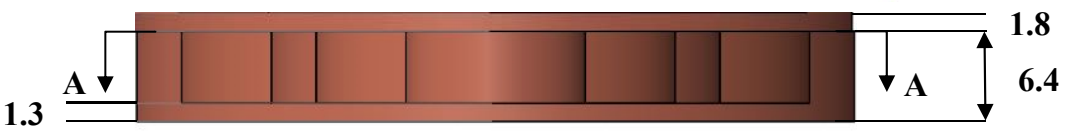

(b)

View A-A

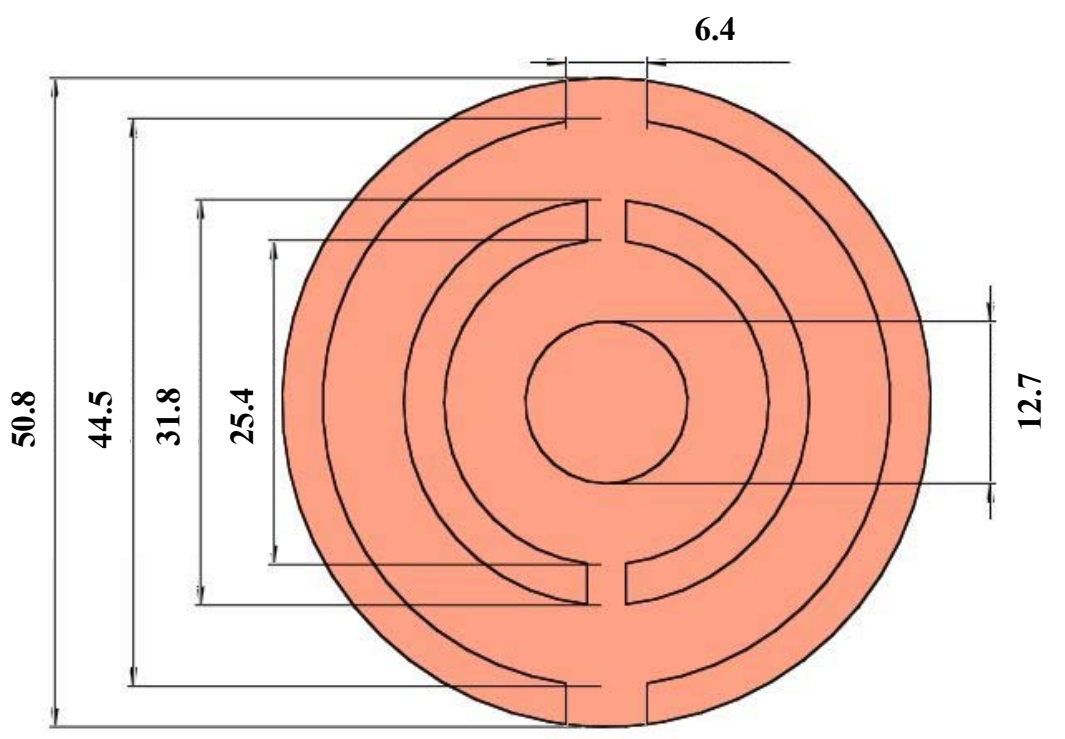

Figure 4: Side (a) and top (b) views of the cold plate with its associated dimensions (all in $\mathrm{mm})$. 


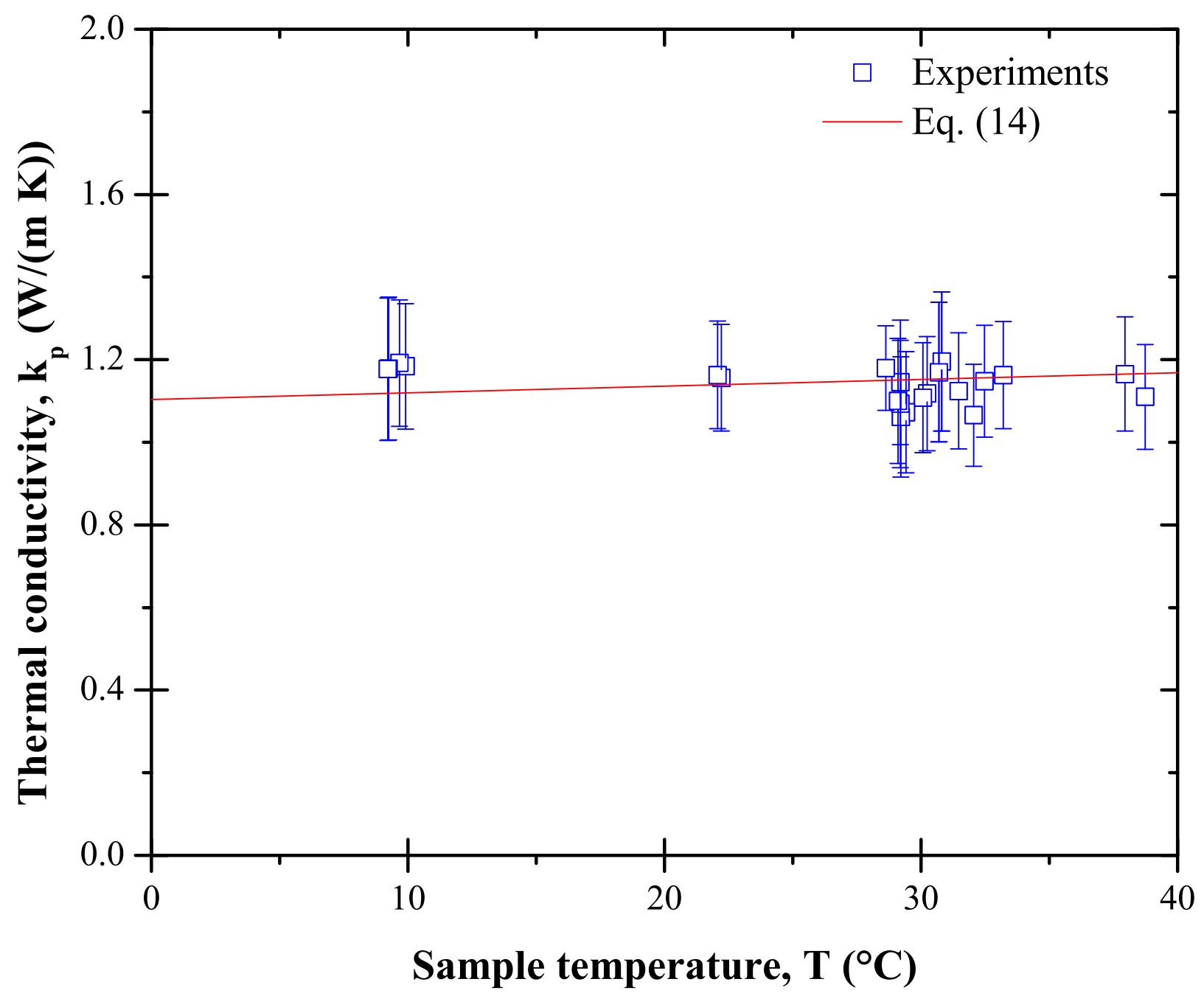

Figure 5: Measured thermal conductivity of Pyrex glass and the reported thermal conductivity of the Pyrex certified reference material BCR-039 given by Equation (15) [57]. 


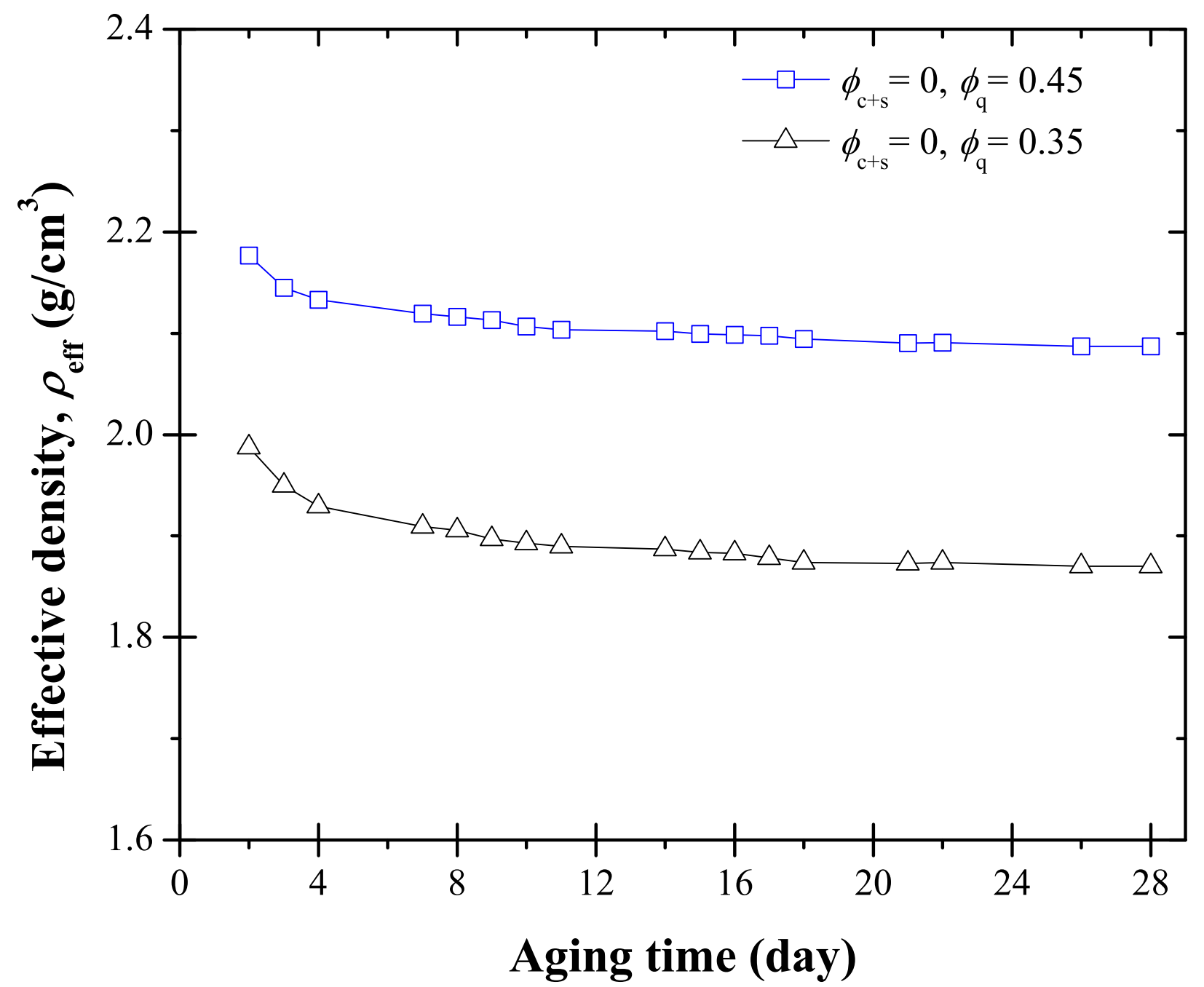

Figure 6: Effective density $r h o_{\text {eff }}$ of cement mortar containing graded quartz sand with volume fraction $\phi_{q}$ of 0.45 and 0.35 but no microencapsulated PCM $\left(\phi_{c+s}=0\right)$ over a 28 day hardening period. 
(a)

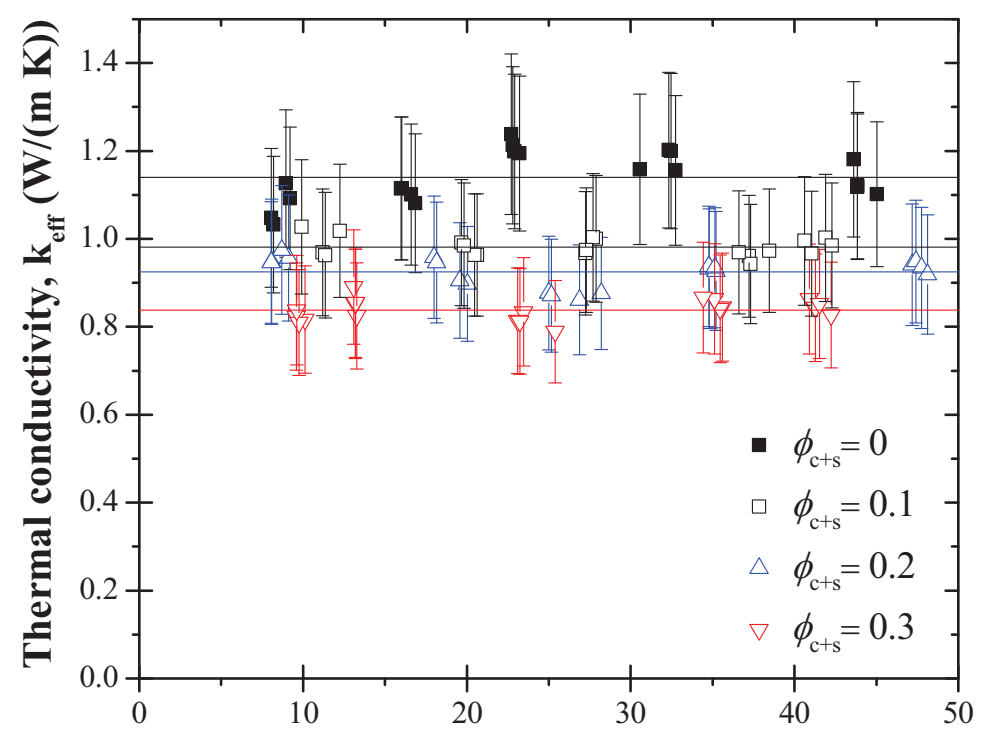

(b)

Sample temperature, $\mathbf{T}\left({ }^{\circ} \mathrm{C}\right)$

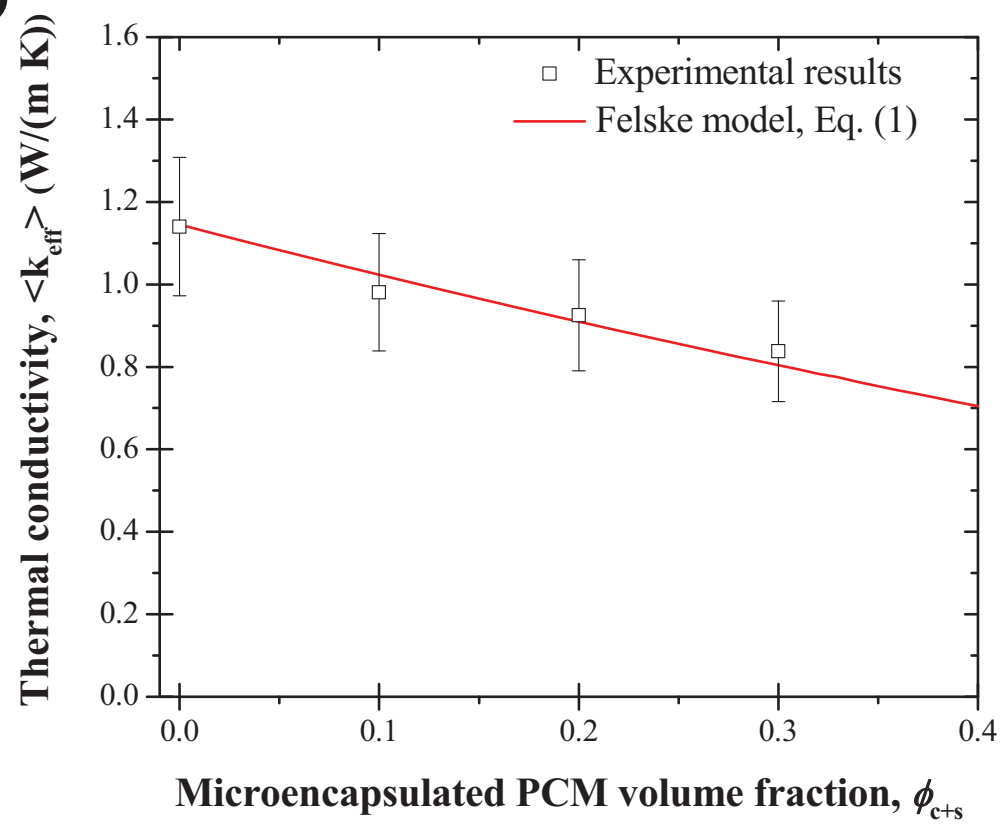

Figure 7: (a) Measured effective thermal conductivity $k_{\text {eff }}$ of cement paste with a constant w/c ratio of 0.45 and volume fraction of microencapsulated PCM $\phi_{c+s}$ between 0 and 0.3 as a function of temperature $T$. (b) Temperature-averaged effective thermal conductivity $<k_{e f f}>$ as a function of $\phi_{c+s}$ with the predictions of the Felske model [38] given by Equation (2). 
(a)

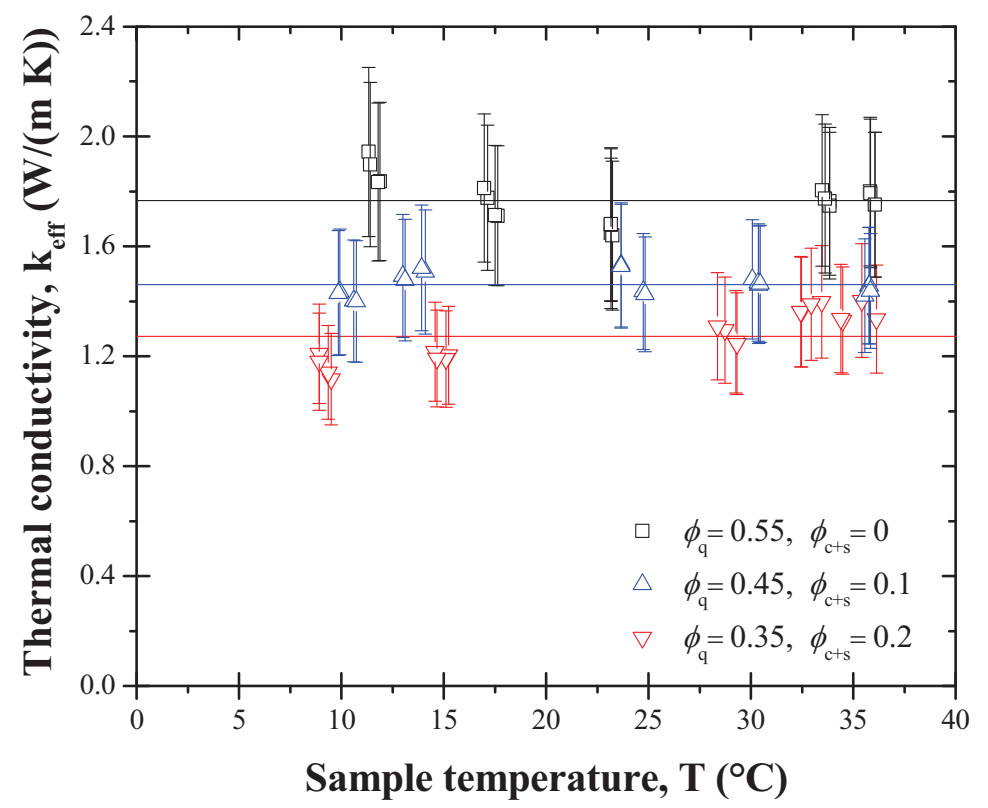

(b)

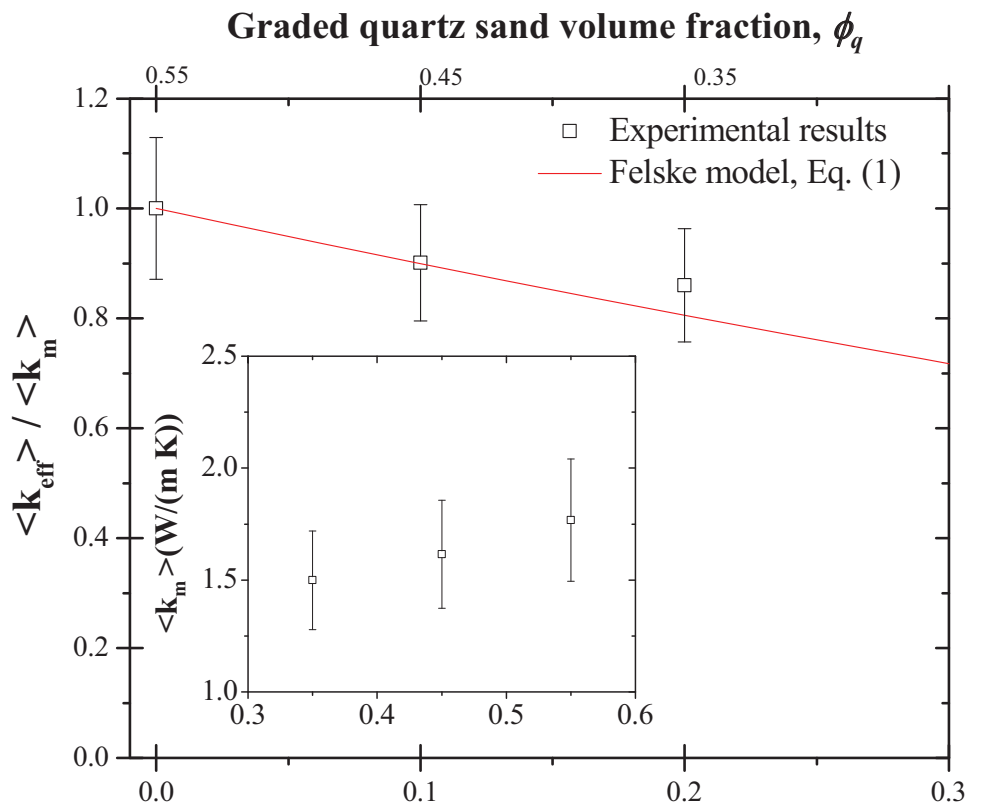

Microencapsulated PCM volume fraction, $\phi_{c+s}$

Figure 8: (a) Measured effective thermal conductivity of cement mortar with a constant w/c of 0.45 with volume fraction of microencapsulated PCM $\phi_{c+s}$ between 0 and 0.3 and graded quartz sand $\phi_{q}$ between 0.35 and 0.55 such that $\phi_{q}+\phi_{c+s}=0.55$ as a functions of temperature $T$. (b) Ratio of measured temperature-averaged thermal conductivity $\left\langle k_{e f f}>/\right.$ $<k_{m}>$ of cement mortar with constant w/c of 0.45 as a function of volume fraction of microencapsulated PCM $\phi_{c+s}$ between 0 and 0.3 for graded quartz sand volume fraction $\phi_{q}$ such that $\phi_{q}+\phi_{c+s}=0.55$. Here, $<k_{m}\left(\phi_{q}\right)>40$ shown in the inset. 
Table 1: Thermal conductivity measurements reported in the literature for cement paste $[23-26,33]$.

\begin{tabular}{ccccccc}
\hline w/c ratio & Temp. $\left({ }^{\circ} \mathbf{C}\right)$ & Age & $\phi_{w}$ & Method & $\begin{array}{c}k_{\text {eff }} \\
(\mathbf{W} /(\mathbf{m} \cdot \mathbf{K}))\end{array}$ & Ref. \\
\hline 0.3 & $20^{\circ} \mathrm{C}$ & 28 days & N/A & TPS & $0.95-1.05$ & {$[23]$} \\
0.4 & $20^{\circ} \mathrm{C}$ & 28 days & N/A & TPS & $0.95-1.05$ & {$[23]$} \\
0.45 & N/A & 28 days & N/A & Laser flash & 0.53 & {$[24]$} \\
0.35 & N/A & 28 days & N/A & Laser flash & 0.53 & {$[25]$} \\
0.35 & N/A & 28 days & 0 & Hot wire & 1.23 & {$[26]$} \\
0.50 & N/A & 1 day & 0.06 & TPS & 1.38 & {$[31]$} \\
0.50 & N/A & 6 days & 0.01 & TPS & 1.21 & {$[31]$} \\
0.40 & $20^{\circ} \mathrm{C}$ & 3 days & 1 & Hot wire & 1.21 & {$[33]$} \\
0.40 & $20^{\circ} \mathrm{C}$ & 7 days & 1 & Hot wire & 1.19 & {$[33]$} \\
0.40 & $20^{\circ} \mathrm{C}$ & 14 days & 1 & Hot wire & 1.20 & {$[33]$} \\
0.40 & $20{ }^{\circ} \mathrm{C}$ & 28 days & 1 & Hot wire & 1.15 & {$[33]$} \\
0.40 & $20{ }^{\circ} \mathrm{C}$ & 28 days & 0 & Hot wire & 0.80 & {$[33]$} \\
0.40 & $60^{\circ} \mathrm{C}$ & 28 days & 1 & Hot wire & 1.10 & {$[33]$} \\
0.40 & $60^{\circ} \mathrm{C}$ & 28 days & 0 & Hot wire & 0.72 & {$[33]$} \\
\hline & & & & & \\
\hline
\end{tabular}

TPS: Transient plane source, N/A: Not specified 
Table 2: Thermal conductivity measurements reported in the literature for cement mortar $[25,28,33]$.

\begin{tabular}{|c|c|c|c|c|c|c|c|}
\hline$\phi_{a g g}$ & $\begin{array}{l}\mathrm{w} / \mathrm{c} \\
\text { ratio }\end{array}$ & $\begin{array}{c}\text { Temp. } \\
\left({ }^{\circ} \mathrm{C}\right)\end{array}$ & Age & $\phi_{w}$ & Method & $\begin{array}{c}k_{e f f} \\
(\mathbf{W} /(\mathbf{m} \cdot \mathbf{K}))\end{array}$ & Ref. \\
\hline N/A (sand) & 0.35 & $\mathrm{~N} / \mathrm{A}$ & 28 days & $\mathrm{N} / \mathrm{A}$ & Laser flash & 0.58 & [25] \\
\hline $0.38^{+}$ & 0.65 & $18-35$ & $\mathrm{~N} / \mathrm{A}$ & 0 & Hot plate & 1.25 & [28] \\
\hline $0.38^{+}$ & 0.65 & $18-35$ & $\mathrm{~N} / \mathrm{A}$ & 1 & Hot plate & 1.94 & [28] \\
\hline $0.3^{-}$ & 0.65 & $18-35$ & $\mathrm{~N} / \mathrm{A}$ & 0 & Hot plate & 1.30 & [28] \\
\hline $0.3^{-}$ & 0.65 & $18-35$ & $\mathrm{~N} / \mathrm{A}$ & 1 & Hot plate & 2.08 & [28] \\
\hline $0.29^{*}$ & 0.65 & $18-35$ & $\mathrm{~N} / \mathrm{A}$ & 0 & Hot plate & 2.56 & [28] \\
\hline $0.29^{*}$ & 0.65 & $18-35$ & $\mathrm{~N} / \mathrm{A}$ & 1 & Hot plate & 4.39 & {$[28]$} \\
\hline $0.7^{s}$ & 0.40 & 20 & 28 days & 1 & Hot wire & 2.47 & [33] \\
\hline $0.7^{s}$ & 0.40 & 40 & 28 days & 1 & Hot wire & 2.50 & [33] \\
\hline $0.7^{s}$ & 0.40 & 60 & 28 days & 1 & Hot wire & 2.30 & [33] \\
\hline $0.7^{s}$ & 0.40 & 20 & 28 days & 0 & Hot wire & 1.97 & [33] \\
\hline $0.7^{s}$ & 0.40 & 40 & 28 days & 0 & Hot wire & 1.94 & [33] \\
\hline $0.7^{s}$ & 0.40 & 60 & 28 days & 0 & Hot wire & 1.84 & [33] \\
\hline $0.35^{s}$ & 0.40 & 20 & 28 days & 1 & Hot wire & 1.71 & [33] \\
\hline $0.35^{s}$ & 0.40 & 40 & 28 days & 1 & Hot wire & 1.74 & [33] \\
\hline $0.35^{s}$ & 0.40 & 60 & 28 days & 1 & Hot wire & 1.64 & [33] \\
\hline $0.35^{s}$ & 0.40 & 20 & 28 days & 0 & Hot wire & 1.28 & [33] \\
\hline $0.35^{s}$ & 0.40 & 40 & 28 days & 0 & Hot wire & 1.27 & [33] \\
\hline $0.35^{s}$ & 0.40 & 60 & 28 days & 0 & Hot wire & 1.15 & [33] \\
\hline $0.25^{p}$ & $\mathrm{~N} / \mathrm{A}$ & $\mathrm{N} / \mathrm{A}$ & 28 days & 0 & Hot wire & 1.35 & {$[34]$} \\
\hline $0.50^{p}$ & $\mathrm{~N} / \mathrm{A}$ & $\mathrm{N} / \mathrm{A}$ & 28 days & 0 & Hot wire & 1.17 & {$[34]$} \\
\hline $0.50^{p}$ & $\mathrm{~N} / \mathrm{A}$ & $\mathrm{N} / \mathrm{A}$ & 28 days & 0 & Hot wire & 1.05 & [34] \\
\hline
\end{tabular}

+ : quartz \& dolerite, ${ }^{-}$: quartz \& barytes, ${ }^{*}$ : quartz \& haematite, ${ }^{s}$ : sand \& stone, ${ }^{p}$ : pumice, N/A: Not specified 
Table 3: Thermal conductivity measurements reported in the literature for cement mortar with impregnated or microencapsulated PCM [36,37].

\begin{tabular}{lccccccccc}
\hline \multirow{2}{*}{ Material } & $\phi_{\text {agg }}^{+}$ & $\phi_{c+s}$ & $\begin{array}{c}\text { w/c } \\
\text { ratio }\end{array}$ & $\begin{array}{c}\text { Temp. } \\
\left({ }^{\circ} \mathbf{C}\right)\end{array}$ & Age & $\phi_{w}$ & Method & $\begin{array}{c}k_{e f f} \\
(\mathbf{W} /(\mathbf{m} \cdot \mathbf{K}))\end{array}$ & Ref. \\
\hline \multirow{3}{*}{ Impregnated } & $0.2^{-}$ & $0^{t}$ & 0.5 & N/A & 28 days & N/A & TPS & 2.06 & {$[36]$} \\
& $0.2^{-}$ & $0.1^{t}$ & 0.5 & N/A & 28 days & N/A & TPS & 1.82 & {$[36]$} \\
& $0.2^{-}$ & $0.2^{t}$ & 0.5 & N/A & 28 days & N/A & TPS & 1.56 & {$[36]$} \\
& $0.2^{-}$ & $0.3^{t}$ & 0.5 & N/A & 28 days & N/A & TPS & 1.35 & {$[36]$} \\
& $0.2^{-}$ & $0^{t}$ & 0.5 & N/A & N/A & N/A & TPS & 2.19 & {$[37]$} \\
& $0.2^{-}$ & $0.012^{t}$ & 0.5 & N/A & N/A & N/A & TPS & 1.97 & {$[37]$} \\
Microencapsulated & $0.2^{-}$ & $0.017^{t}$ & 0.5 & N/A & N/A & N/A & TPS & 1.90 & {$[37]$} \\
& $0.2^{-}$ & $0.025^{t}$ & 0.5 & N/A & N/A & N/A & TPS & 1.85 & {$[37]$} \\
\hline & 0.63 & 0 & 0.45 & $19-28^{\circ} \mathrm{C}$ & 28 days & N/A & Hot wire & 3.4 & {$[8]$} \\
& 0.57 & 0.025 & 0.45 & $19-28^{\circ} \mathrm{C}$ & 28 days & N/A & Hot wire & 2.9 & {$[8]$} \\
& 0.57 & 0.124 & 0.45 & $19-28^{\circ} \mathrm{C}$ & 28 days & N/A & Hot wire & 2.1 & {$[8]$} \\
\hline
\end{tabular}

${ }^{+}$: Non PCM aggregates, ${ }^{t}$ : Mass fraction (volume fraction not given), TPS: Transient plane source N/A: Not specified 


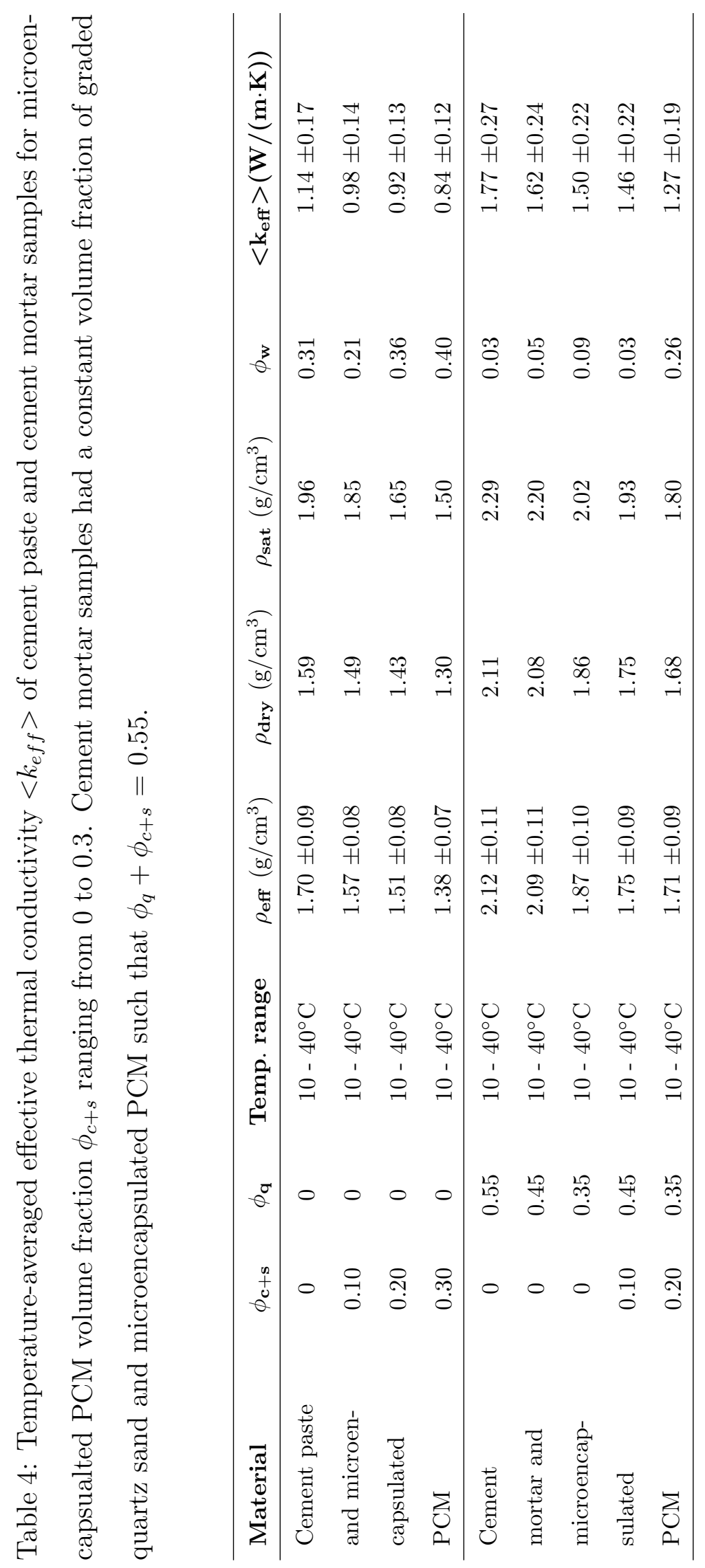

